\title{
Nitric oxide-sensitive guanylyl cyclase stimulation improves experimental heart failure with preserved ejection fraction
}

\author{
Nicola Wilck, ${ }^{1,2,3,4}$ Lajos Markó, ${ }^{1,2,3,5}$ András Balogh, ${ }^{1,2,3,4}$ Kristin Kräker, ${ }^{1,2,3,5}$ Florian Herse, ${ }^{1,2,5}$ \\ Hendrik Bartolomaeus, ${ }^{1,3}$ István A. Szijártó, ${ }^{1,4}$ Maik Gollasch,, ${ }^{1,3,4}$ Nadine Reichhart, ${ }^{4}$ Olaf Strauss, ${ }^{4}$ \\ Arnd Heuser, ${ }^{5}$ Damian Brockschnieder, ${ }^{6}$ Axel Kretschmer, ${ }^{6}$ Ralf Lesche, ${ }^{6}$ Florian Sohler, ${ }^{6}$ \\ Johannes-Peter Stasch, ${ }^{6}$ Peter Sandner, ${ }^{6}$ Friedrich C. Luft, , ${ }^{1,2,4,5}$ Dominik N. Müller, ${ }^{1,2,3,4,5}$ \\ Ralf Dechend, ${ }^{1,2,4,7}$ and Nadine Haase $e^{1,2,3,5}$ \\ 'Experimental and Clinical Research Center, Max-Delbrück Center for Molecular Medicine and the Charité Medical Faculty, \\ Berlin, Germany. ${ }^{2}$ Berlin Institute of Health (BIH), Berlin, Germany. ${ }^{3}$ DZHK (German Centre for Cardiovascular Research), \\ Berlin, Germany. ${ }^{4}$ Charité - Universitätsmedizin Berlin, Freie Universität Berlin, Humboldt-Universität zu Berlin, and BIH, \\ Berlin, Germany. ${ }^{5}$ Max-Delbrück Center for Molecular Medicine, Berlin, Germany. ${ }^{6}$ Bayer AG, Drug Discovery, Wuppertal \& \\ Berlin, Germany. ${ }^{7}$ HELIOS-Klinikum, Berlin, Germany.
}

Heart failure with preserved ejection fraction (HFpEF) can arise from cardiac and vascular remodeling processes following long-lasting hypertension. Efficacy of common HF therapeutics is unsatisfactory in HFpEF. Evidence suggests that stimulators of the nitric oxide-sensitive soluble guanylyl cyclase (NOsCC) could be of use here. We aimed to characterize the complex cardiovascular effects of NOsCC stimulation using NO-independent stimulator BAY 41-8543 in a double-transgenic rat (dTCR) model of HFpEF. We show a drastically improved survival rate of treated dTCR. We observed less cardiac fibrosis, macrophage infiltration, and gap junction remodeling in treated dTCR. Microarray analysis revealed that treatment of dTCR corrected the dysregulateion of cardiac genes associated with fibrosis, inflammation, apoptosis, oxidative stress, and ion channel function toward an expression profile similar to healthy controls. Treatment reduced systemic blood pressure levels and improved endothelium-dependent vasorelaxation of resistance vessels. Further comprehensive in vivo phenotyping showed an improved diastolic cardiac function, improved hemodynamics, and less susceptibility to ventricular arrhythmias. Short-term BAY 418543 application in isolated untreated transgenic hearts with structural remodeling significantly reduced the occurrence of ventricular arrhythmias, suggesting a direct nongenomic role of NOsGC stimulation on excitation. Thus, NOsCC stimulation was highly effective in improving several HFpEF facets in this animal model, underscoring its potential value for patients.

Authorship note: RD and NH are co-senior authors.

Conflict of interest: DB, JPS, and PS are coinventors in several patent applications on soluble guanylyl cyclase agonists and are employees of Bayer AG. AK, RL, and FS are employees of Bayer AG.

Submitted: June 28, 2017 Accepted: January 11, 2018 Published: February 22, 2018

Reference information: JCI Insight. 2018;3(4):e96006. https://doi.org/10.1172/jci. insight.96006.

\section{Introduction}

The diagnosis and treatment of chronic heart failure are challenging (1). By determining the ejection fraction (EF), 2 main entities may be distinguished: heart failure with reduced $\mathrm{EF}$ (HFrEF) or heart failure with preserved EF (HFpEF) (2). HFpEF is characterized by diastolic dysfunction and concentric left ventricular (LV) remodeling due to alterations in myocardial structure, function, and intramyocardial signaling (3). As a result, both the extracellular matrix and myofilaments are affected so that ventricular stiffness is increased $(4,5)$. Additionally, oxidative stress, inflammation, and endothelial dysfunction play a role in $\operatorname{HFpEF}(2,6)$. HFpEF is associated with advancing age and comorbidities such as hypertension and diabetes $(1,7)$. Up to $90 \%$ of HFpEF patients are hypertensive $(8,9)$. Dysregulation of the renin-angiotensin-aldosterone system (RAAS) plays an important role in hypertension, mainly due to its effector peptide, angiotensin II (Ang II) $(10,11)$. The RAAS is commonly activated in patients with HFpEF (12). Nonetheless, RAAS-directed therapy has not been shown to be effective $(8,13,14)$. In contrast to HFrEF, no pharmacological therapy has been shown to improve the outcomes in $\operatorname{HFpEF}(9,15-17)$.

The nitric oxide (NO)/cyclic GMP (cGMP)/PKG pathway represents a potentially novel and promising therapeutic target for $\operatorname{HFpEF}(18,19)$. Oxidative stress, inflammation, and endothelial dysfunction 
in HFpEF lead to decreased levels of cGMP and reduced PKG activity (20). Reduction of cGMP synthesis as a consequence of diminished NO bioavailability is followed by deficient NO-sensitive or soluble guanylyl cyclase (NOsGC) stimulation. The NO/cGMP pathway is pivotal for cardiac and vascular function (20-23). NOsGC stimulators, which are independent of NO production, offer a potentially novel approach to increase cGMP levels. Such stimulators are currently under investigation as a potential treatment for HFpEF $(19,24)$. With riociguat, the first NOsGC stimulator has been approved for the treatment of pulmonary arterial hypertension (PAH) and chronic thromboembolic pulmonary hypertension (CTEPH) in 2013 (25).

The limited availability of HFpEF rodent models has impeded mechanistic studies investigating the efficacy of potential treatments (26). We have shown earlier that double-transgenic rats (dTGR) harboring the human renin and angiotensinogen genes are a suitable experimental HFpEF model (27-31). Untreated dTGR die between 7-8 weeks of age from severe heart failure with diastolic dysfunction and preserved EF as a result of a severe systemic hypertension, cardiac hypertrophy with fibrosis and inflammation, electrical remodeling with increased susceptibility to cardiac arrhythmias, cardiac cachexia, pulmonary congestion, and endothelial dysfunction (27-32). Similar to HFpEF patients $(18,20,33)$, dTGR exhibit cGMP deficiency at terminal disease stages (Supplemental Figure 1; supplemental material available online with this article; https://doi.org/10.1172/jci.insight.96006DS1). We used this model to test whether or not NOsGC stimulation with BAY 41-8543, a potent and selective NOsGC stimulator, can ameliorate experimental HFpEF.

\section{Results}

NOSGC stimulation reduces blood pressure and improves endothelial function and survival. BAY 41-8543 significantly increased serum and myocardial cGMP levels compared with vehicle-treated dTGR (serum, 60.2 \pm 4.2 vs. $39.1 \pm 7.9 \mathrm{pmol} / \mathrm{ml}, P=0.02$; myocardial, $15.9 \pm 1.6$ vs. $11.3 \pm 1.2 \mathrm{pmol} / \mathrm{ml}, P=0.04)$. Furthermore, BAY 41-8543 increased survival to $100 \%$ at week 7 , no different than nontransgenic Sprague Dawley (SD) control rats. In contrast, $75 \%$ of vehicle-treated dTGR died (Figure 1A). BAY 41-8543 treatment prevented cardiac cachexia in dTGR (Figure 1B). Systolic pressure increased progressively in vehicle-treated dTGR from $169 \pm 4 \mathrm{mmHg}$ at week 5 to $197 \pm 11 \mathrm{mmHg}$ at week 7 , as measured by tail cuff. BAY 41-8543 treatment significantly reduced systolic blood pressure to $133 \pm 4 \mathrm{mmHg}$ at week 7 , whereas $\mathrm{SD}$ rats were normotensive at $89 \pm 4 \mathrm{mmHg}$ (Figure $1 \mathrm{C}$ ). To evaluate blood pressure kinetics 24 hours after BAY 41-8543 administration at weeks 5-7, blood pressure monitoring was performed using radiotelemetry (Figure 1D and Supplemental Figure 3, A-C). Blood pressure reduction was consistent over the study course and most pronounced at week 7 (Supplemental Figure 3, A-C). Blood pressure reduction was consistently observed approximately 1 hour after administration and ceased after 12-14 hours (Supplemental Figure 3, A-C), leading to a significant net blood pressure reduction at week 7 (Figure 1D).

We examined the effect of BAY 41-8543 treatment on endothelial function of resistance vessels. Mesenteric arteries were isolated from 7-week-old vehicle- and BAY 41-8543-treated dTGR. Twenty-four hours after the last treatment, when serum cGMP levels were no longer elevated (data not shown), vasorelaxation was tested in a wire myograph. Dose-response curves to acetylcholine (Ach) showed an improved endothelium-dependent vasorelaxation of arteries isolated from BAY 41-8543-treated dTGR (Figure 1E). Endothelium-independent relaxation of mesenteric arteries to sodium nitroprusside was unchanged in BAY 41-8543-treated compared with vehicle-treated dTGR (Supplemental Figure 4), suggesting an improved function of the vascular endothelium.

NOsGC stimulation improves cardiac gene expression without altering myocardial hypertrophy. We next investigated the impact of BAY 41-8543 on cardiac hypertrophy. Compared with SD, dTGR showed considerable cardiac hypertrophy. Despite blood pressure reduction and improvement of endothelial function, vehicle-treated and BAY 41-8543-treated dTGR had similar cardiac hypertrophy indices (heart/ tibia ratio, Figure 2A) and cardiomyocyte sizes (Figure 2B). Echocardiography confirmed these findings, since interventricular septal thickness (IVSd, Figure 2C) and LV posterior wall thickness at end-diastole (LVPWd, Figure 2D) as well as LV mass (data not shown) were significantly increased in vehicle-treated dTGR compared with SD controls but were unchanged with BAY 41-8543. We observed the characteristic gene expression shift for cardiac $\alpha$-myosin heavy chain (Mhy6) toward the fetal $\beta$-myosin heavy chain (Mhy7) isoform in vehicle-treated dTGR compared with SD controls (Figure 2E). BAY 41-8543 treatment prevented the shift toward the fetal $\beta$-myosin heavy chain (Mhy7) isoform (Figure 2F), indicating 

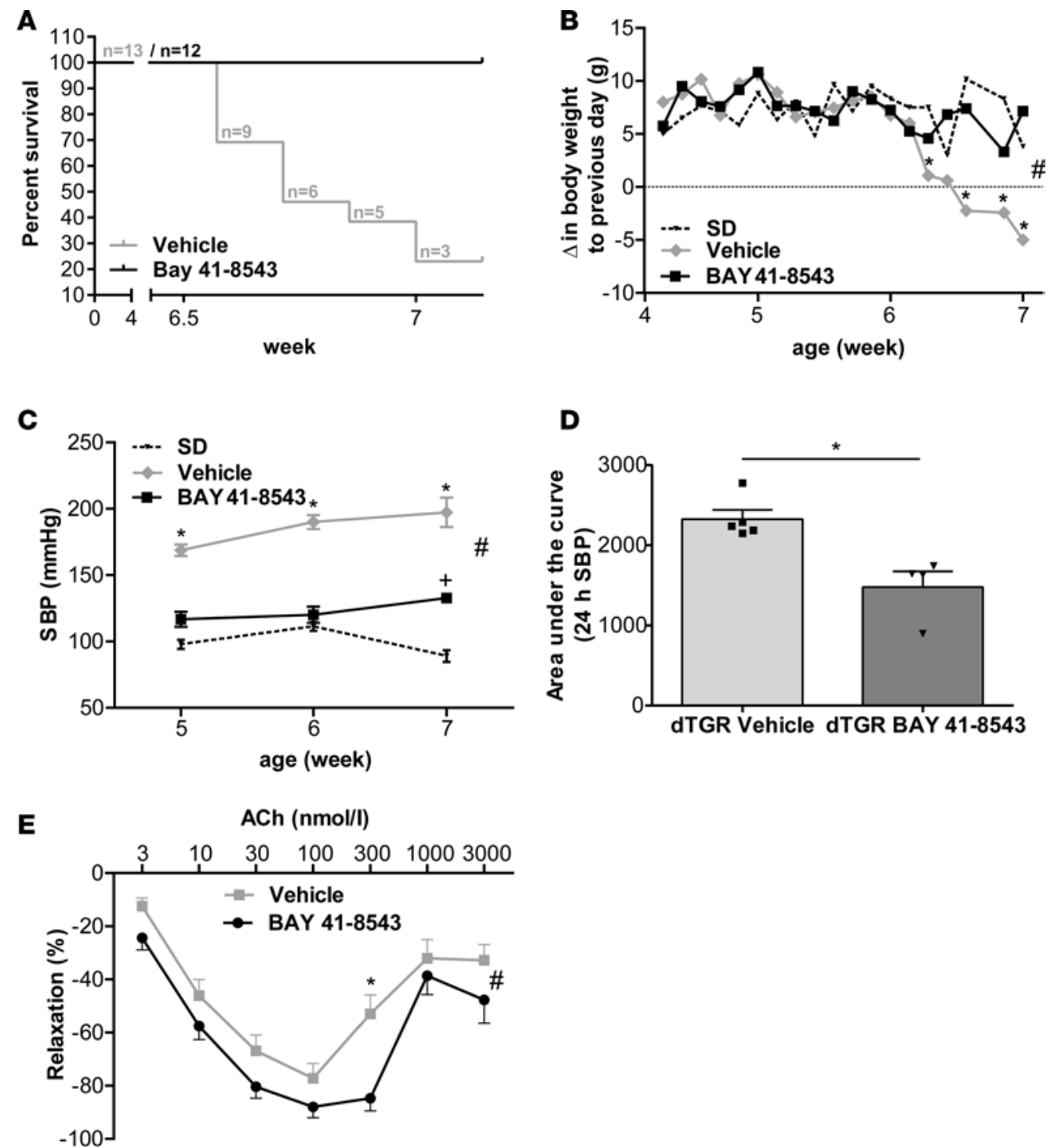

Figure 1. NOsGC stimulation reduces blood pressure and improves endothelial function and survival. (A) Kaplan-Meier survival analysis of vehicle-treated dTCR and dTCR receiving BAY 41-8543. At week 7, only 24\% of vehicle-treated dTCR survived. BAY 41-8543 improved the survival of the dTCR to $100 \%$. Survival curves are statistically different by the log-rank test $(P<0.0001)$. No SD rats died before end of study (data not shown). (B) Rats were weighed daily. Vehicle-treated dTCR lost weight significantly from week 6 to week 7. In SD rats and BAY 41-8543-treated dTCR, no change in weight was observed ( ${ }^{*} P<0.05$, between all groups, 2 -way ANOVA with Bonferroni post hoc test; ${ }^{*} P<0.05$ vehicle vs. BAY $41-8543$ and SD, Bonferroni post hoc test; data expressed as mean \pm SEM; SD, $n=6$; vehicle, $n=4-13$; BAY 41-8543, $n=12$ ). (C) Tail-cuff systolic blood pressure (SBP) at weeks 5, 6, and 7. SBP increased in vehicle-treated dTCR. BAY 41-8543 significantly reduced SBP. SD rats were normotensive $\left({ }^{\sharp} P<0.05,{ }^{*} P<0.01\right.$ vehicle vs. BAY $41-8543$ and SD, ${ }^{+} P<$ 0.01 BAY 41-8543 vs. SD; data expressed as mean \pm SEM; 2-way ANOVA with Bonferroni post hoc test; SD, $n=6$; vehicle, $n=6$; BAY 41-8543, $n=12$ ). (D) Radiometric telemetry SBP over 24 hours on day 21 of treatment. Data are expressed as AUC in vehicle- and BAY 41-8543-treated dTCR. BAY 41-8543 significantly reduced SBP ( ${ }^{*} P<0.05$; Mann Whitney $U$ test; data expressed as mean \pm SEM; vehicle, $n=5$; BAY 41-8543, $\left.n=4\right)$. (E) Myograph analysis on mesenteric arteries of dTCR showed in the presence of acetylcholine improved endothelial function in BAY 41-8543-treated rats compared with vehicle-treated dTCR in a dose-dependent manner $\left({ }^{*} P<0.05,{ }^{*} P<0.05\right.$ vehicle vs. BAY 41-8543; data expressed as mean \pm SEM; 2 -way ANOVA with Bonferroni post hoc test; vehicle, $n=31$; BAY 41-8543, $n=30$ ).

that — despite a similar degree of hypertrophy — myocardial remodeling might be improved compared with vehicle-treated dTGR.

Hypertension-induced cardiac remodeling in dTGR is characterized by a global dysregulation of cardiac genes (31). Principle-component analysis revealed 2 distinct clusters of cardiac gene expression (Figure 3A). One cluster was composed entirely of SD controls (green circles). BAY 41-8543- and vehicle-treated dTGR were allocated to 2 additional clusters. All BAY 41-8543-treated animals (blue squares) were restricted to 1 cluster, whereas vehicle-treated dTGR (red diamonds) were part of both. 
A
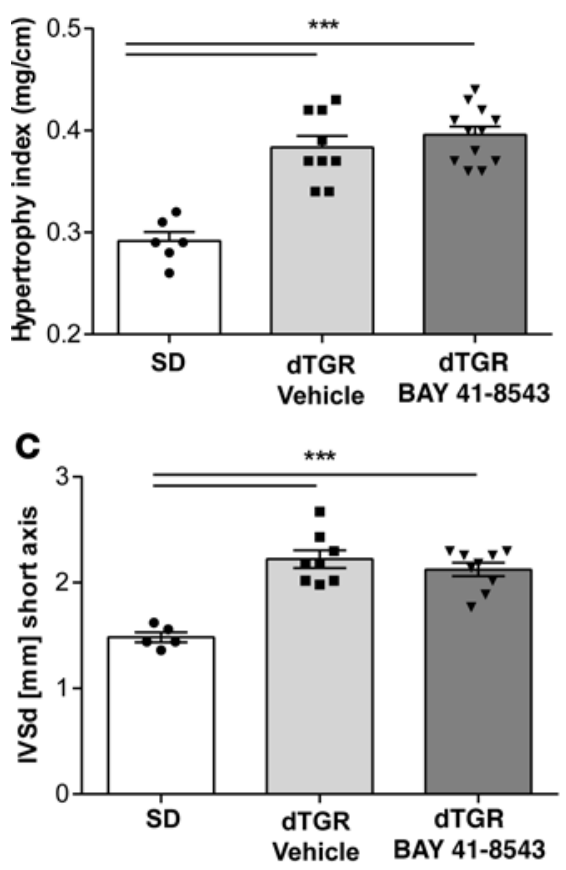

B

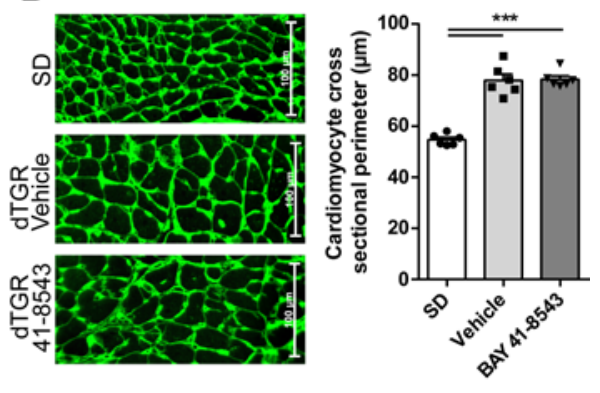

D

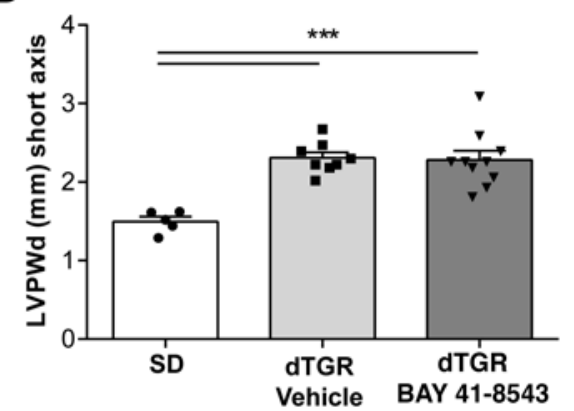

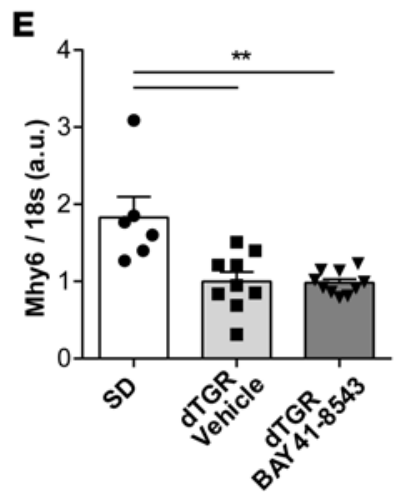

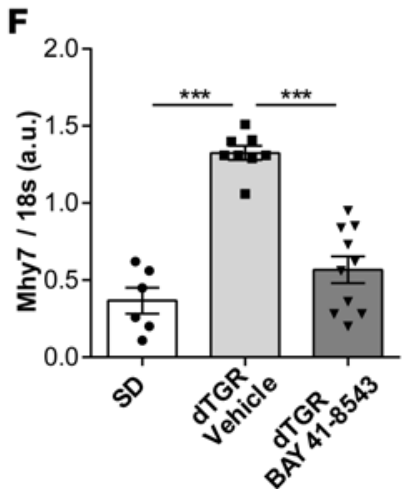

Figure 2. Myocardial hypertrophy remains unaltered after NOsGC stimulation. (A) Hypertrophy index expressed as heart weight to tibia length was increased in vehicle-treated dTCR compared with SD rats. Treatment with BAY 41-8543 did not attenuate the hypertrophy index ( ${ }^{* * *} P<0.001 ; 1$-way ANOVA with Tukey's post hoc test; data expressed as mean \pm SEM; SD, $n=6$; vehicle, $n=9$; BAY 41-8543, $n=12$ ). (B) Cardiomyocyte size was examined by immunohistological analysis of WGA-stained heart sections. Analysis of cardiomyocyte cross sectional perimeter revealed increased values in vehicle-treated dTCR compared with SD rats, whereas no effect of BAY 41-8543 treatment on the size of the cardiomyocytes was observed (*** $P<0.001$; 1-way ANOVA with Tukey's post hoc test; data expressed as mean \pm SEM; SD, $n=6$; vehicle, $n=6$; BAY 41-8543, $n=6$ ). (C) Interventricular septal thickness in end diastole (IVSd) was increased in vehicle- and BAY 41-8543-treated dTGR compared with SD rats $\left({ }^{* * *} P<0.001\right.$; 1-way ANOVA with Tukey's post hoc test; data expressed as mean \pm SEM; SD, $n=5$; vehicle, $n$ $=8$; BAY 41-8543, $n=9$ ). (D) Left ventricular diastolic posterior wall thickness (LVPWd) was elevated in vehicle- and BAY 41-8543-treated dTCR compared with SD rats ( ${ }^{* *} P<0.001$; 1-way ANOVA with Tukey's post hoc test; data expressed as mean \pm SEM; SD, $n=5$; vehicle,$n=8$; BAY 41-8543, $n=10$ ). (E) Cardiac mRNA expression of $\alpha$ myosin heavy chain (Mhy6) was significantly lower in vehicle- and BAY 41-8543-treated dTCR compared with SD rats. There were no differences in mRNA expression between vehicle- and BAY 41-8543-treated dTCR ( ${ }^{* *} P<0.01 ; 1$-way ANOVA with Tukey's post hoc test; data expressed as mean \pm SEM; SD, $n=6$; vehicle, $n=9$; BAY 41-8543, $n=10$ ). (F) Cardiac mRNA expression of $\beta$ myosin heavy chain (Mhy7) was significant higher in vehicle-treated dTCR compared with SD rats. BAY 41-8543 treatment led to a reduction in mRNA expression compared with vehicle ${ }^{* * *} P<0.001 ; 1$-way ANOVA with Tukey's post hoc test; data expressed as mean \pm SEM; SD, $n=6$; vehicle, $n=8$; BAY 41-8543, $n=10$ ).

In previous studies, we could demonstrate that dTGR with terminal heart failure showed an altered expression profile compared with preterminal dTGR (31). In the present study, cardiac gene expression analysis showed 607 genes to be significantly dysregulated in vehicle-treated dTGR compared with SD. Of those dysregulated genes, 248 were reversed upon BAY 41-8543 treatment (Figure 3B). Two-way hierarchical clustering of the 844 genes whose expression markedly changed between BAY 41-8543 and vehicle-treated dTGR (Figure 3B) revealed a similar clustering as for the principal component analysis (Supplemental Figure 5). 
A

A
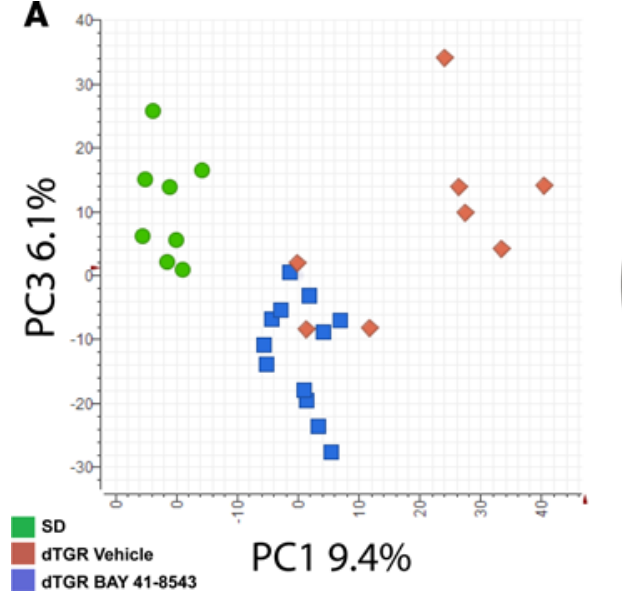

B

reversal upon BAY 41-8543 treatment

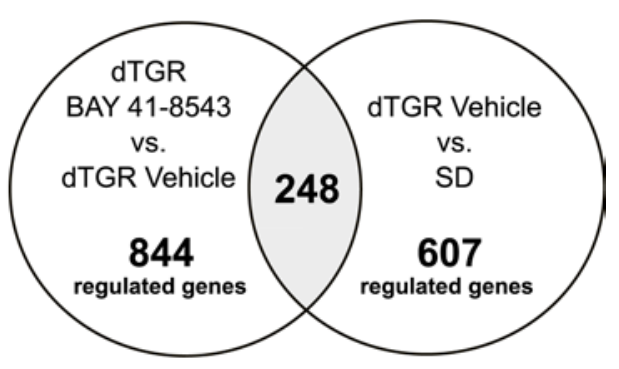

Figure 3. NOsGC stimulation restores dysregulated cardiac gene expression. (A) Principle-component analysis (PCA) of the cardiac microarray original data. Each dot corresponds to 1 rat; green circles for SD rats, red diamonds for vehicle-treated dTCR, and blue squares for BAY 41-8543-treated dTCR. PCA showed 2 clusters (SD, $n=8$; vehicle, $n$ $=8$; BAY 41-8543, $n=12$ ). (B) Venn diagram of differentially expressed cardiac genes in BAY 41-8543-treated versus vehicle-treated $\mathrm{dTCR}$ and vehicle-treated $\mathrm{dTCR}$ versus SD rats.

Of note, genes associated with gene ontology terms such as fibrosis, inflammation, apoptosis, oxidative stress, and ion channel function were significantly attenuated by BAY 41-8543 treatment and showed a reversal of dysregulated genes in dTGR by BAY 41-8543 treatment (Supplemental Figure 6 , A-F, and Supplemental Figure 7, A-F). Along the same line, BAY 41-8543 improved several heart failure serum biomarkers. Serum brain natriuretic peptide (BNP), osteopontin, and heart-type fatty acid binding protein (H-FABP) were significantly reduced in BAY 41-8543-treated dTGR (Supplemental Figure 8).

NOsGC stimulation improves diastolic function and enhances cardiac performance, despite unaltered myocardial hypertrophy. Further evidence for an improved cardiac function is derived from detailed echocardiographic and hemodynamic analyses. Although slightly lower than SD controls, LV EF and fractional shortening (FS) were preserved in vehicle-treated dTGR. BAY 41-8543 treatment led to a significant increase in EF and FS compared with vehicle-treated dTGR (Figure 4A and Table 1).

Mitral flow velocity Doppler was used to determine LV diastolic function. The ratio of peak velocities of early to late mitral inflow (E/A ratio) was elevated in vehicle-treated dTGR compared with SD controls (Figure 4B), suggesting a restrictive filling pattern. In line with this, vehicle-treated dTGR displayed a significantly reduced deceleration time (DTT, Figure 4C) and a significantly increased isovolumetric relaxation time (IVRT) compared with SD controls, altogether indicative of an impaired LV relaxation. In BAY 41-8543-treated dTGR, E/A ratio, DTT, and IVRT were normalized toward SD values, suggesting an improvement of LV filling (Figure 4, B-D).

Two-dimensional (2-D) speckle tracking echocardiography-derived (STE-derived) measurement of LV strain and strain rate was performed to further examine LV myocardial performance. Global longitudinal strain (Figure 4E), global circumferential strain (Figure 4F), and global radial strain (Figure 4G) were significantly reduced in vehicle-treated dTGR compared with SD controls and improved by BAY 41-8543. Similar results were observed for the respective global strain rates (data not shown). To assess regional LV dyssynchrony, the standard deviation of time-to-peak strain was measured (Figure $4 \mathrm{H}$ and Supplemental Figure 9). Time-to-peak circumferential (Figure 4I) and radial (Figure 4J) strains were significantly delayed in vehicle-treated dTGR compared with SD controls and were normalized by BAY 41-8543 treatment. No significant alterations in time-to-peak longitudinal strain were observed across all groups (Supplemental Figure 9). Taken together, different echocardiography approaches suggest an improved LV systolic and diastolic performance in BAY 41-8543-treated dTGR.

Cardiac catheterization was performed to obtain detailed analysis of the cardiac hemodynamics. LV pressure-volume (PV) loops from vehicle-treated dTGR showed remarkable alterations characteristic of HFpEF (Figure 5A). LVESP was increased in vehicle-treated dTGR compared with SD controls and was reduced in 
A

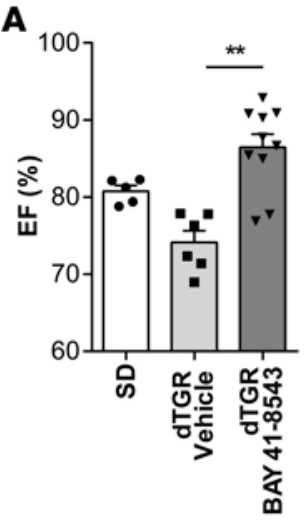

E

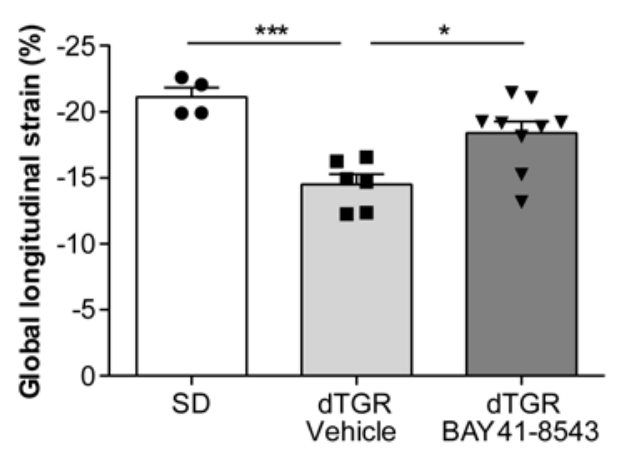

B

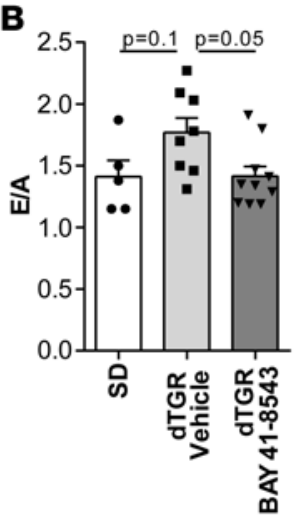

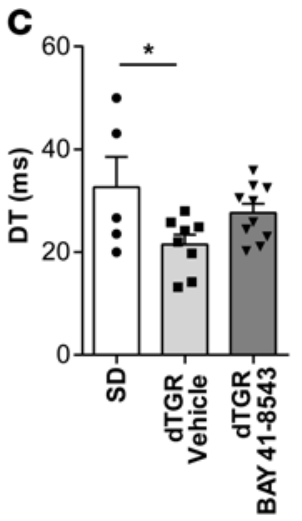

D

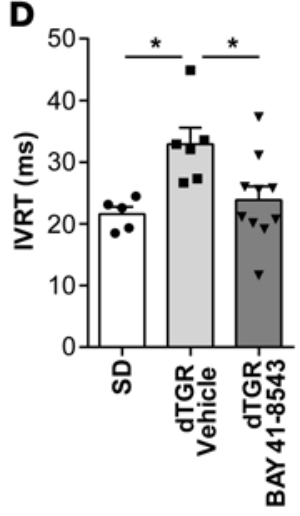

$\mathbf{F}$

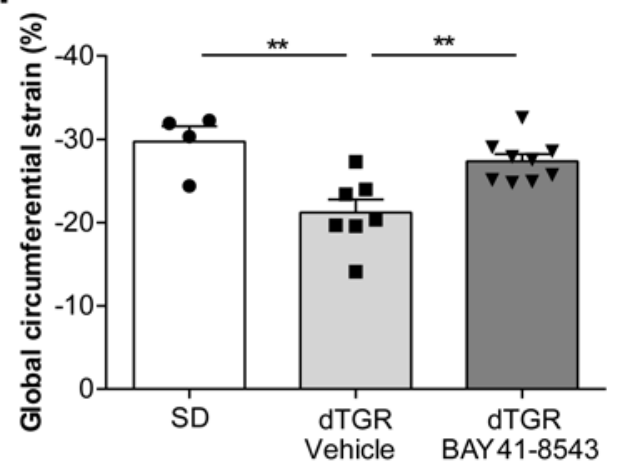

G

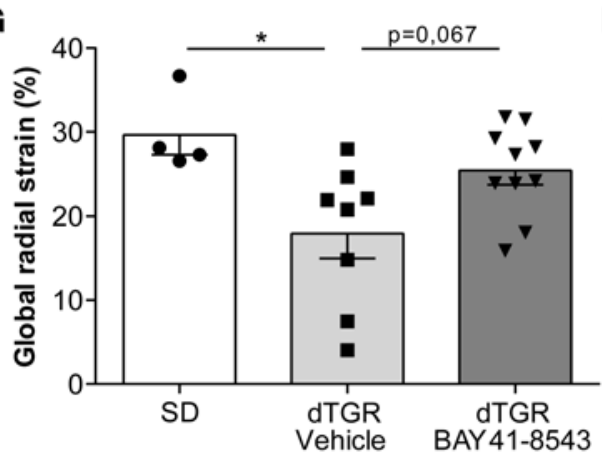

H

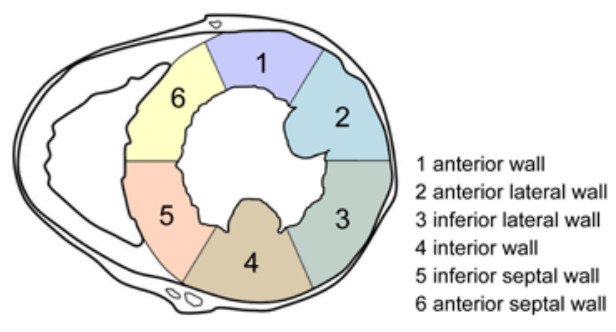

I
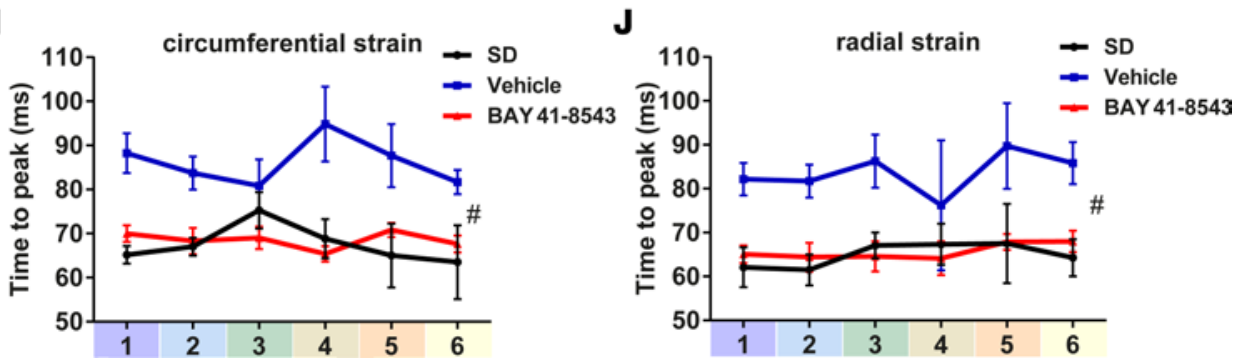

Figure 4. Stimulation of NOsGC improves diastolic function and regional diastolic compliance. (A) Ejection fraction (EF) values of the left ventricle were preserved in vehicle-treated dTCR. BAY 41-8543 administration led to a significant increase in EF (** $P<0.01 ; 1$-way ANOVA with Dunn's post hoc test; data expressed as mean \pm SEM; SD, $n=5$; vehicle, $n=6$; BAY 41-8543, $n=10$ ). (B) Ratio of peak E velocity to peak A velocity (E/A) was slightly but not significantly increased in vehicle-treated dTGRs $(P=0.1)$.The E/A ratio was reduced by BAY 41-8543 treatment $(P=0.05 ; 1$-way ANOVA with Tukey's post hoc test; data expressed as mean $\pm \mathrm{SEM}$; SD, $n=5$; vehicle, $n=8$; BAY 41-8543, $n=10$ ). (C) Mitral valve E-wave deceleration time (DTT) was shortened in vehicle-treated dTCR ( ${ }^{*} P<0.05$; 1-way ANOVA with Tukey's post hoc test; data expressed as mean \pm SEM; SD, $n=5 ;$ vehicle, $n=8 ;$ BAY 41-8543, $n=10$ ). (D) Isovolumetric relaxation time (IVRT) was prolonged in vehicle-treated dTCRs. Administration of BAY 41-8543 diminished the IVRT ( $P<0.05$; 1-way ANOVA with Tukey's post hoc test; data expressed as mean \pm SEM; SD, $n=5$; vehicle, $n=6$; BAY 41-8543, $n=10$ ). (E) Global longitudinal strain (GLS) was reduced in vehicle-treated dTCR. BAY 41-8543 treatment led to an increase in GLS $\left({ }^{*} P<0.05 ;{ }^{* *} P<0.001 ; 1\right.$-way ANOVA with Tukey's post hoc test; data expressed as mean \pm SEM; SD, $n=4$; vehicle, $n=6$; BAY 41-8543, $n=9$ ). (F) Global circumferential strain (GCS) was decreased in vehicle-treated dTCR. BAY 41-8543 treatment led to an increase in GCS $\left({ }^{* *} P<0.01 ; 1\right.$-way ANOVA with Tukey's post hoc test; data expressed as mean \pm SEM; $\mathrm{SD}, n=4$; vehicle, $n=7$; BAY 41-8543, $n=9$ ). (C) Global radial strain (GRS) was lower in vehicle-treated dTGR. BAY 41-8543 treatment led to an increase in 
GRS compared with vehicle but did not reach $(P=0.07)$ significance $\left({ }^{*} P<0.05\right.$; 1 -way ANOVA with Tukey's post hoc test; data expressed as mean \pm SEM, SD, $n=4$; vehicle, $n=8$; BAY 41-8543, $n=10$ ). (H) For assessment of regional myocardial strain in the parasternal short axis, the left ventricle was divided into 6 segments. (I) Time-to-peak circumferential strain is elevated in vehicle-treated dTCR. BAY 41-8543 administration led to a shorter time-to-peak strain in circumferential axis ( ${ }^{P} P<0.5$; 2-way ANOVA; data expressed as mean $\pm \mathrm{SEM}$; SD, $n=4$; vehicle, $n=6$; BAY 41-8543, $n=10$ ). (J) Time-to-peak radial strain was elevated in vehicle-treated dTCR. BAY 41-8543 administration led to a shorter time-to-peak strain in radial axis ( $P<0.5 ; 2$-way ANOVA; data expressed as mean \pm SEM; SD, $n=4$; vehicle, $n=6$; BAY 41-8543, $n=10$ ).

BAY 41-8543-treated dTGR (Table 2). LVEDP was moderately elevated in all dTGR groups compared with SD controls, albeit not reaching significance $(8.48 \pm 0.96 \mathrm{mmHg} \mathrm{SD}, 10.91 \pm 1.80$ vehicle-treated dTGR, $10.38 \pm 1.07$ BAY 41-8543-treated dTGR). Significant changes in LV end systolic volume (LVESV) and LV end diastolic volume (LVEDV) were not observed between vehicle-treated, BAY 41-8543-treated dTGR and SD controls (Table 2). Predicted end-diastolic PV relationship (EDPVR) compliance curves (34) showed a leftward/upward shift in vehicle-treated dTGR. BAY 41-8543 treatment led to a rightward/downward shift in EDPVR toward the slope of SD controls (Figure 5B). Cardiac index was elevated in BAY 41-8543-treated dTGR compared with vehicle-treated dTGR and SD control (Figure 5C). Moreover, BAY 41-8543 normalized total peripheral resistance (TPR) and arterial volume elastance (Ea) to SD levels, while being increased in vehicle-treated dTGR (Figure 5, D and E). Taken together, echocardiographic and hemodynamic examinations indicate impaired LV diastolic function with preserved systolic function in vehicle-treated dTGR and a remarkable improvement of diastolic function upon BAY 41-8543 treatment.

NOSGC stimulation ameliorates cardiac inflammation and fibrosis. Cardiac dysfunction in failing hearts is typically associated with myocardial inflammation and fibrosis. Administration of BAY 41-8543 led to a reduction of cardiac macrophage and monocyte infiltration compared with vehicle-treated dTGR (Figure 6A). Furthermore, chronic BAY 41-8543 treatment resulted in a significant reduction of perivascular matrix deposition (Figure 6B), as well as interstitial cardiac fibrosis (Figure 6C), which was supported by the normalization of the profibrotic genes connective tissue growth factor $(\operatorname{Ctg} f)$ and neutrophil gelatinase-associated lipocalin (Ngal; Figure 6D).

NOSGC stimulation improves electrical remodeling and rhythm disturbances. Alongside enhanced ventricular structural remodeling, dTGR display enhanced vulnerability to ventricular arrhythmias (27). To analyze the effect of NOsGC stimulators on rhythm disturbances and electrical remodeling, we performed in vivo electrophysiological investigations. Programmed electrical stimulation demonstrated a significantly reduced vulnerability to ventricular arrhythmias in BAY 41-8543-treated dTGR compared with vehicle-treated dTGR (Figure 7, A and B). BAY 41-8543 treatment significantly reduced the ventricular tachycardia induction rate from $46 \% \pm 12 \%$ in vehicle-treated dTGR to $11 \% \pm 7 \%$ (Figure $7 \mathrm{~B}$ ). Similarly, induced tachycardias longer than 10 consecutive beats were reduced to $6 \% \pm 6 \%$ in BAY $41-8543-$ treated dTGR compared with $41 \% \pm 14 \%$ in vehicle-treated dTGR (Figure 7C).

Since gap junctional remodeling predisposes to arrhythmic disturbances, we analyzed the LV for alterations in the intracellular localization of connexin 43 (Cx43), the major ventricular gap junction protein, by immunofluorescence. In healthy SD hearts, $\mathrm{Cx} 43$ was expressed at the intercalated disc while being abnormally located to the lateral surfaces in dTGR hearts. BAY 41-8543 treatment relocated $\mathrm{Cx} 43$ expression to the intercalated disc, indicating protection from gap junctional remodeling (Supplemental Figure 10).

To evaluate whether or not NOsGC stimulation has antiarrhythmogenic properties in structurally remodeled hearts, we performed ECG recordings of Langendorff-perfused hearts isolated from 7-weekold untreated dTGR. For this, occurrence of spontaneous ventricular extrasystoles (VES) was analyzed in isolated perfused dTGR hearts acutely infused with $3 \mu \mathrm{M}$ BAY 41-8543 or vehicle (DMSO). BAY 41-8543infused dTGR hearts exhibited significantly less VES compared with solvent-treated dTGR hearts (Figure 7D). BAY 41-8543 reduced VES/minute in a time-dependent manner, with a reciprocal increase in solvent-treated dTGR hearts (Figure 7E). These data indicate that activation of NO/cGMP pathway may have antiarrhythmogenic effects, despite preexisting structural remodeling.

\section{Discussion}

The major finding in our study is that NOsGC stimulation sharply reduces mortality and morbidity in an experimental HFpEF model. Surprisingly and despite substantial blood pressure reduction, the cardiac hypertrophy was not reduced. NOsGC stimulation ameliorated cardiac fibrosis and inflammation, improved 
Table 1. Echocardiographical analysis

\begin{tabular}{|c|c|c|c|c|c|c|}
\hline & & & & $P$ value & $P$ value & $P$ value \\
\hline & SD & dTGR vehicle & dTGR BAY 41-8543 & SD vs dTGR vehicle & $\begin{array}{c}\text { SD vs dTGR BAY } \\
41-8543\end{array}$ & $\begin{array}{l}\text { dTCR vehicle vs. } \\
\text { dTGR BAY 41-8543 }\end{array}$ \\
\hline $\begin{array}{c}\text { Fractional } \\
\text { shortening (\%) }\end{array}$ & $49.14 \pm 0.8717$ & $39.52 \pm 4.413$ & $57.59 \pm 2.409$ & 0.7575 & 0.2699 & $0.0026^{A}$ \\
\hline$E(\mathrm{~mm} / \mathrm{s})$ & $871.9 \pm 67.13$ & $827.6 \pm 65.93$ & $896.4 \pm 33.34$ & 0.8584 & 0.9506 & 0.594 \\
\hline$A(\mathrm{~mm} / \mathrm{s})$ & $646.7 \pm 84.64$ & $491.8 \pm 63.71$ & $646.9 \pm 34.57$ & 0.2093 & 0.9999 & 0.1122 \\
\hline Ejection time (ms) & $52.75 \pm 5.827$ & $54.63 \pm 6.223$ & $50.63 \pm 2.557$ & 0.9657 & 0.9533 & 0.7981 \\
\hline Stroke volume ( $\mu \mathrm{l})$ & $170.8 \pm 13.27$ & $127.7 \pm 9.963$ & $145.1 \pm 11.86$ & 0.0885 & 0.3438 & 0.5398 \\
\hline $\begin{array}{l}\text { Cardiac output (ml/ } \\
\text { min) }\end{array}$ & $65.27 \pm 4.622$ & $49.72 \pm 3.654$ & $59.96 \pm 5.757$ & 0.1845 & 0.7831 & 0.3423 \\
\hline Heart rate (bpm) & $383.4 \pm 9.7$ & $407.6 \pm 28.0$ & $409.9 \pm 9.1$ & 0.6936 & 0.9953 & 0.624 \\
\hline LVd (mm) short axis & $6.36 \pm 0.10$ & $6.21 \pm 0.19$ & $6.35 \pm 0.29$ & 0.9185 & 0.9986 & 0.9096 \\
\hline $\begin{array}{l}\text { LV sys (mm) short } \\
\text { axis }\end{array}$ & $3.234 \pm 0.04$ & $3.77 \pm 0.32$ & $2.72 \pm 0.23$ & 0.4151 & 0.4192 & $0.017^{\mathrm{B}}$ \\
\hline $\begin{array}{c}\text { Mitral valve ejection } \\
\text { time (ms) }\end{array}$ & $52.75 \pm 5.83$ & $54.63 \pm 6.22$ & $50.63 \pm 2.56$ & 0.9657 & 0.9533 & 0.7981 \\
\hline$E^{\prime}(\mathrm{mm} / \mathrm{s})$ & $59.3 \pm 1.548$ & $51.68 \pm 6.872$ & $56.33 \pm 4.459$ & 0.7005 & 0.9429 & 0.8001 \\
\hline$A^{\prime}(\mathrm{mm} / \mathrm{s})$ & $39.95 \pm 1.049$ & $36.41 \pm 6.770$ & $48.40 \pm 4.269$ & 0.9205 & 0.6113 & 0.2322 \\
\hline$E / E^{\prime}$ & $14.81 \pm 1.205$ & $17.50 \pm 2.160$ & $16.74 \pm 1.315$ & 0.6346 & 0.7776 & 0.9387 \\
\hline Tei index & $0.748 \pm 0.131$ & $0.891 \pm 0.169$ & $0.792 \pm 0.058$ & 0.7365 & 0.9688 & 0.808 \\
\hline
\end{tabular}

Tei index = (IVCT + IVRT)/ET where IVCT represents isovolumic contraction time, IVRT represents isovolumic relaxation time, and ET represents ejection time. ${ }^{A} P<0.01,{ }^{B} P<0.05,{ }^{C} P<0.001$, 1-way ANOVA with Tukey's post hoc test. E, peak velocity of early mitral inflow; $A$, peak velocity of late mitral inflow; LVPWsys, left ventricular posterior wall thickness at end systole; LVd, left ventricular end-diastolic diameter; LVsys, left ventricular end-systolic diameter; E', early diastolic mitral annular velocity; A', late diastolic mitral annular velocity.

endothelium-dependent vasorelaxation and cardiac electrical remodeling, and reversed dysregulated cardiac genes even tough myocardial hypertrophy persisted. The beneficial action of BAY 41-8543 consisted of improved diastolic function, increased regional diastolic compliance, and enhanced cardiac performance.

Currently, no treatment has yet been shown to reduce morbidity and mortality in HFpEF patients. RAAS blockers were not effective in reducing morbidity and mortality $(8,13,14)$. The management is limited to ameliorating symptoms with diuretics and the treatment of comorbidities, such as hypertension, diabetes, and atrial fibrillation.

Our results indicate that cardiac hypertrophy does not solely contribute to HFpEF development, and our findings emphasize the role of cardiac fibrosis and microvascular inflammation. Less recruitment of immune cells to cardiac tissue and vessels, less cardiac fibrosis and electrical remodeling, and improved endothelial function are major contributors to the reduced mortality and morbidity in our experimental HFpEF model. A similarly improved survival was shown for HFpEF in Dahl salt-sensitive rats, where treatment with cardiosphere-derived cells reduced cardiac inflammation and fibrosis despite persisting cardiac hypertrophy (35). Taken together, BAY 41-8543 treatment reversed proinflammatory and profibrotic stimuli and thereby prevented the development of HFpEF. We found no changes in cardiac hypertrophy comparing vehicle and BAY 41-8543-treated rats assessed by hypertrophy index, cardiomyocyte cross-sectional perimeter, and echocardiography. Similar to the findings of Gallet et al., we postulate that reduction of cardiac hypertrophy is not necessarily required to substantially improve diastolic function (35).

Activation, recruitment, and transendothelial migration of leukocytes and macrophages into the heart leading to LV fibrosis have been shown in humans (36) and in our experimental HFpEF model $(27-29,31)$. Investigators have proposed that the diverse comorbidities of HFpEF contribute to a systemic inflammatory state, which induces microvascular endothelial inflammation resulting in endothelial dysfunction, ROS 
Table 2. Pressure-volume conductance catheter analysis

\begin{tabular}{|c|c|c|c|c|c|c|}
\hline & & & & $P$ value & $P$ value & $P$ value \\
\hline & SD & dTGR vehicle & dTCR BAY 41-8543 & SD vs. dTGR vehicle & $\begin{array}{c}\text { SD vs. dTGR BAY } \\
41-8543\end{array}$ & $\begin{array}{l}\text { dTGR vehicle vs. } \\
\text { dTGR BAY 41-8543 }\end{array}$ \\
\hline MAP (mmHg) & $100.7 \pm 1.619$ & $163.0 \pm 14.68$ & $107.1 \pm 13,86$ & 0.0376 & 0.9545 & $0.0199^{A}$ \\
\hline LVESP (mmHg) & $100.7 \pm 1.619$ & $163.0 \pm 14.68$ & $107.1 \pm 13.86$ & 0.0376 & 0.9545 & $0.0199^{A}$ \\
\hline LVEDP (mmHg) & $8.475 \pm 0.9578$ & $10.91 \pm 1.799$ & $10.38 \pm 1.073$ & 0.6638 & 0.6638 & 0.7757 \\
\hline LVESV $(\mu \mathrm{l})$ & $23.37 \pm 1.974$ & $36.37 \pm 10.42$ & $30.01 \pm 5.835$ & 0.7400 & 0.7943 & 0.7943 \\
\hline Pmax (mmHg) & $106.2 \pm 1.09$ & $172.0 \pm 13.47$ & $130.8 \pm 12.04$ & $0.0132^{c}$ & 0.4381 & 0.0557 \\
\hline Pmin (mmHg) & $3.90 \pm 1.38$ & $7.70 \pm 1.69$ & $6.56 \pm 0.65$ & 0.1691 & 0.3706 & 0.76 \\
\hline Pmean (mmHg) & $49.65 \pm 0.25$ & $73.4 \pm 4.39$ & $59.00 \pm 6.34$ & 0.05 & 0.5546 & 0.1594 \\
\hline Pdev (mmHg) & $102.3 \pm 2.20$ & $164.3 \pm 13.98$ & $124.3 \pm 11.54$ & $0.0188^{\mathrm{C}}$ & 0.511 & 0.0625 \\
\hline Stroke volume $(\mu \mathrm{l})$ & $184.0 \pm 85.06$ & $75.3 \pm 13.63$ & $130.6 \pm 15.66$ & 0.1369 & 0.5448 & 0.4336 \\
\hline Preload (mmHg) & $9.38 \pm 1.48$ & $6.60 \pm 0.91$ & $6.03 \pm 0.90$ & 0.2439 & 0.1193 & 0.9058 \\
\hline $\begin{array}{l}\text {-dP/dt min } \\
(\mathrm{mmHg} / \mathrm{s})\end{array}$ & $7,287 \pm 476.7$ & $9,239 \pm 962.3$ & $7,783 \pm 1,021$ & 0.4753 & 0.9468 & 0.5251 \\
\hline $\begin{array}{l}\mathrm{dP} / \mathrm{dt} \max \\
(\mathrm{mmHg} / \mathrm{s})\end{array}$ & $6,633 \pm 132.4$ & $9,020 \pm 570.4$ & $8,697 \pm 997.0$ & 0.2344 & 0.3015 & 0.9560 \\
\hline$\tau$ & $12.41 \pm 0.62$ & $12.14 \pm 0.61$ & $10.01 \pm 0.41$ & 0,9495 & $0,0257^{\complement}$ & $0,0179^{c}$ \\
\hline
\end{tabular}

MAP, mean arterial pressure; LVESP, left ventricular end-systolic pressure; LVEDP, left ventricular end-diastolic pressure; LVESV, left ventricular endsystolic volume; LVEDV, left ventricular end-diastolic volume; Pmax, maximum pressure during a loop; Pmin, minimum pressure during a loop; Pmean, average pressure during a loop; Pdev, Pmax - Pmin; LVEDR, left ventricular end-diastolic radius; LVPW, left ventricular posterior wall thickness; Preload, $\left(\right.$ LVEDP $\times$ LVEDR) $/ 2 \times$ LVPW. ${ }^{A} P<0.01,{ }^{B} P<0.001,{ }^{C} P<0.05,{ }^{D} P<0.0001,1$-way ANOVA with Tukey's post hoc test.

production, and reduced NO bioavailability (37). This state of affairs leads to disturbed cGMP signaling. Indeed, cGMP deficiency has been shown to cause HFpEF-associated characteristics, such as diastolic dysfunction, increased LV and peripheral vascular stiffness, and disturbances in cardiac rhythm $(2,18)$. We showed that BAY 41-8543 reduces monocyte/macrophage infiltration into cardiac tissue and reverses inflammatory gene expression patterns in the failing rat hearts. Antiinflammatory properties of NOsGC stimulation has been previously shown by others (38). Cardiac fibrosis is the main contributor to diastolic impairment in $\mathrm{HFpEF}$ as it increases LV and peripheral vascular stiffness $(4,5,39)$. BAY 41-8543 treatment in our model reduced perivascular and interstitial cardiac fibrosis. This finding was paralleled by a beneficial modulation of cardiac gene expression of fibronectin $(F n)$, connected tissue growth factor ( $C t g f)$, and neutrophil gelatinase-associated lipocalin $(\mathrm{Ngal})$. $\mathrm{Ngal}$ has been proposed as a biotarget for cardiovascular fibrosis (40). These results are consistent with other studies using NOsGC stimulators, showing a reduction in cardiac fibrosis in a blood pressure-independent manner $(41,42)$.

Assessment of cardiac function by conventional echocardiography and STE showed an improvement of diastolic function and regional compliance in BAY 41-8543-treated compared with vehicle-treated rats. STE-based analysis of cardiac function has been shown to be more sensitive compared with conventional echocardiography (43). Using conventional echocardiography in our rat model, we merely detected a trend toward an alteration of the E/A ratio. Evaluation of E/A and DTT in rats with a substantially higher heart rate than in humans is difficult; normal values have not been established, and translation into the human situation has to be done with caution. However, the more sensitive STE-based analyses revealed significant alterations in LV global strains and strain rates in our HFpEF model. Santos et al. could show that HFpEF is associated with greater LV mechanical dyssynchrony compared with healthy controls (44). BAY 41-8543 treatment led to an amelioration of LV dyssynchrony in our experimental HFpEF model. Moreover, invasive LV PV measurements confirmed the enhancement of cardiac performance, showing an improved LV filling pattern in BAY 41-8543-treated rats. In combination with the 
A

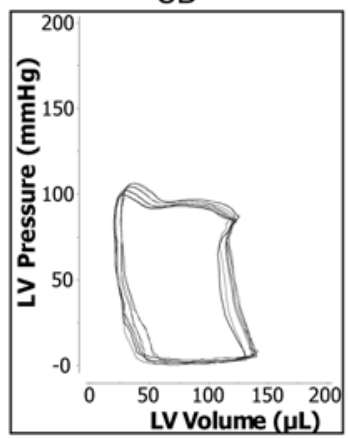

C

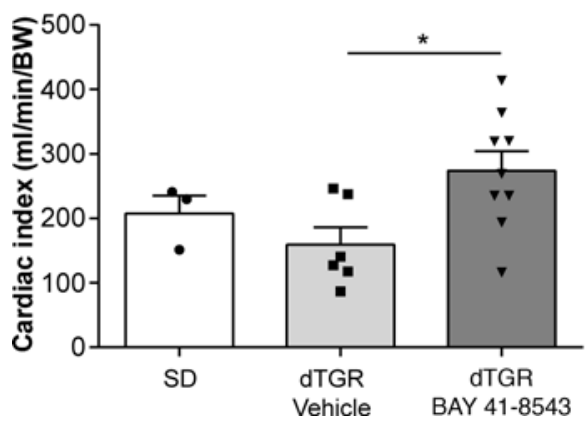

dTGR Vehicle

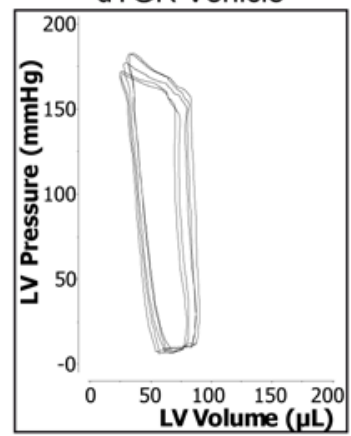

D

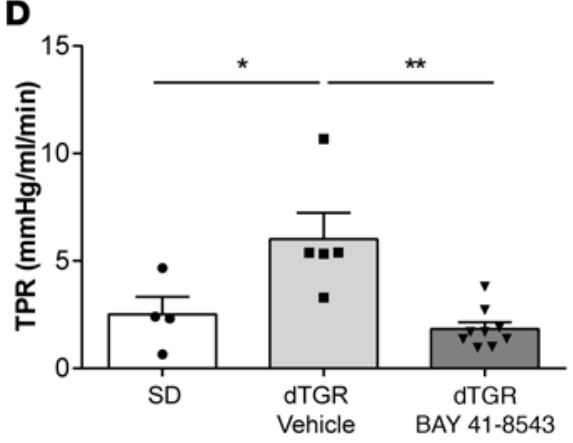

dTGR BAY 41-8543

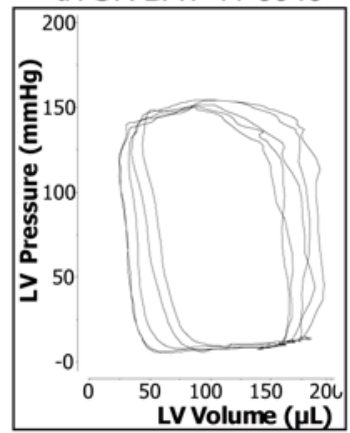

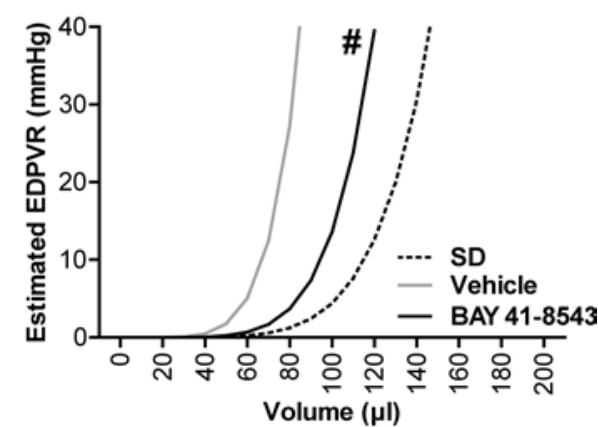

E

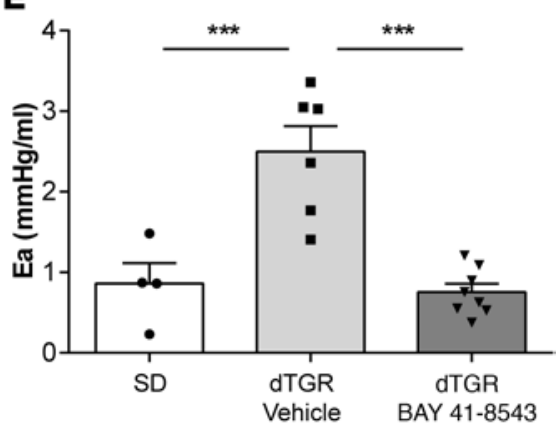

Figure 5. Stimulation of NOsGC enhances cardiac performance in the failing heart. (A) Original recordings of LV pressure-volume loops (P-V) obtained with the Millar P-V conductance catheter system from 1 representative rat from SD group, vehicle, and BAY 41-8543 treated dTCR. (B) Estimated end-diastolic P-V relationship (EDPVR) is shifted leftward in vehicle-treated dTCR compared with SD rats. Treatment with BAY 41-8543 resulted in a rightward shift of EDPVR ( $P<$ 0.5; 2-way ANOVA; data expressed as mean \pm SEM; SD, $n=4$; vehicle, $n=6$; BAY 41-8543, $n=10$ ). (C) Cardiac index was comparable among SD and vehicle-treated dTCR. BAY 41-8543 administration led to an increase in cardiac index $\left({ }^{*} P<0.05 ; 1\right.$-way ANOVA with Tukey's post hoc test; data expressed as mean \pm SEM; SD, $n=3$; vehicle, $n=6$; BAY 41-8543, $n=10$ ). (D) A significantly higher total peripheral resistance (TPR) was observed in vehicle-treated dTCR in comparison with SD rats. BAY 41-8543 reduced the elevated TPR ( ${ }^{*} P<0.05,{ }^{*} P<0.01 ; 1$-way ANOVA with Tukey's post hoc test; data expressed as mean \pm SEM; SD, $n=4$; vehicle, $n=5$; BAY 41-8543, $n=9$ ). (E) Vehicle-treated dTGR showed a significant rise in arterial volume elastance (Ea) compared with SD rats. BAY 41-8543 showed a significant fall of these increases ( ${ }^{* *} P<0.001$; 1-way ANOVA with Tukey's post hoc test; data expressed as mean \pm SEM; SD, $n=4$; vehicle, $n=6$; BAY 41-8543, $n=9$ ).

STE-based analysis and the invasive measurements, we show that BAY 41-8543 is successfully ameliorating diastolic dysfunction.

Cardiac arrhythmias are a hallmark of HFpEF in humans (45) and were also shown earlier in our model (27). BAY 41-8543 administration led to an improved electrical remodeling and a reduced inducibility of ventricular tachycardias despite persistent cardiac hypertrophy. In a previous study, we showed that cardiac hypertrophy is not the sole determinant for increased arrhythmias (27). Improved electrical remodeling following BAY 41-8543 treatment was accompanied by a reversal of ion channel gene expression patterns in the failing rat hearts. Moreover, by using isolated dTGR hearts in ex vivo Langendorff experiments, we demonstrated that BAY 41-8543 effectively reduces the occurrence of VES in hearts with existing structural remodeling. Together, these findings suggest that targeting the NO/cGMP pathway may beneficially address electrical remodeling. In our model, oral administration of BAY 41-8543 resulted in a blood pressure decrease lasting approximately 12 hours. Despite these acute effects, BAY 41-8543 is beneficial on a broad range of HFpEF symptoms. It is known that BAY 41-8543 has a short half-life in rodents (24). NOsGC stimulators with improved pharmacokinetics have been developed for clinical use. Vericiguat, a long-acting sGC stimulator, was applied once a day in HFrEF patients, was well tolerated, and dose-dependently reduced NT-pro BNP levels (trial SOCRATES-REDUCED) (24). Currently, a phase III clinical trial with vericiguat in HFrEF patients is ongoing (ClinicalTrials.gov, NCT 02861534). Of note, vericiguat has just recently been tested in HFpEF patients in the SOCRATES-PRESERVED trial, a phase IIb study investigating tolerability and the optimal dose regimen. Therein, NOsGC stimulation with vericiguat for 12 weeks improved quality of life without changing N-terminal pro-brain natriuretic peptide (NT-proBNP) levels, thus encouraging further studies in HFpEF patients using higher doses and longer follow-up (46). 
A

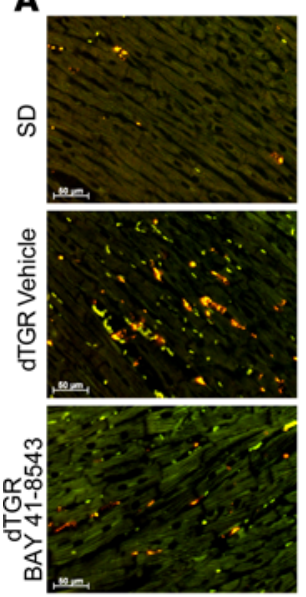

C

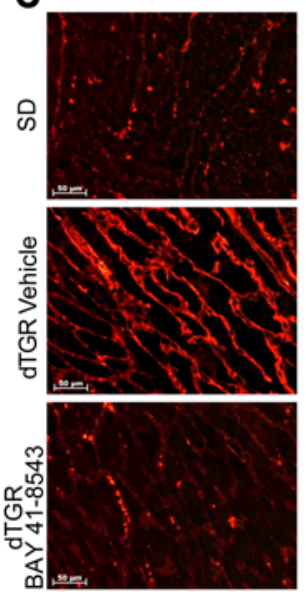

B

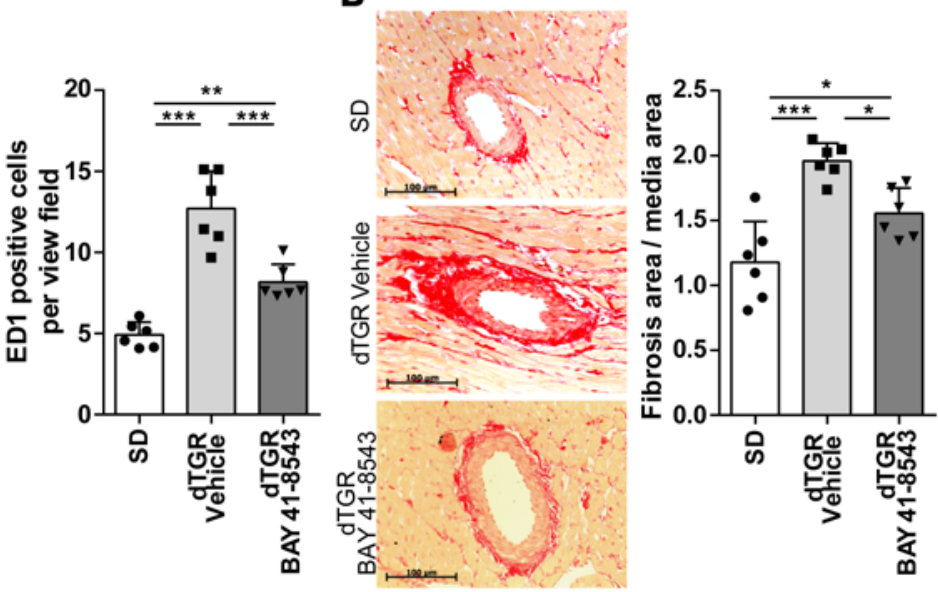

D
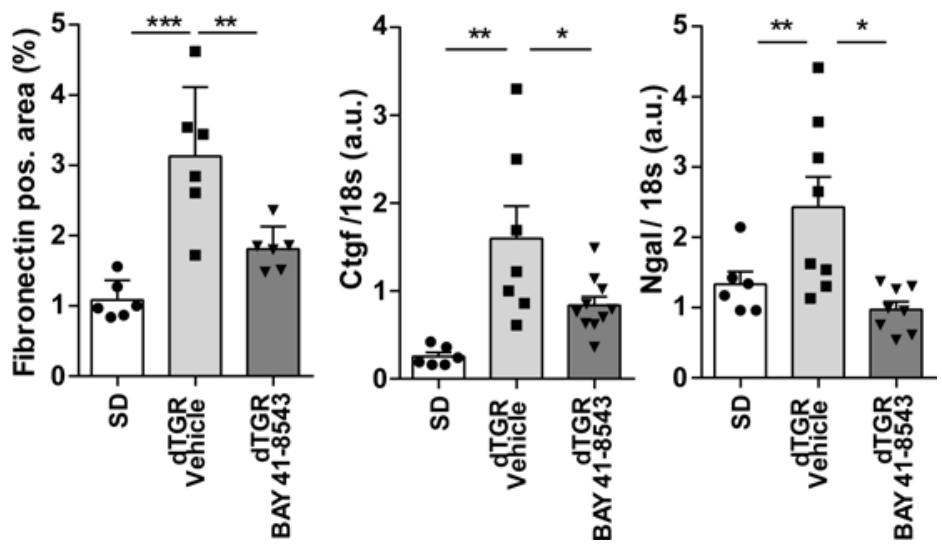

Figure 6. Stimulation of NOsGC ameliorates cardiac inflammation and fibrosis. (A) Representative image of ED-1 IHC staining for macrophages in the heart from SD rats and vehicle- and BAY 41-8543-treated dTCR. Compared with SD controls, the number of macrophages was increased in the heart of vehicle-treated dTGR. This increase was reduced in dTCR administered BAY 41-8543 ( ${ }^{* *} P<0.01$, ${ }^{* * *} P<0.001 ; 1$-way ANOVA with Dunn's post hoc test; data expressed as mean \pm SEM; SD, $n=6$; vehicle, $n=6$; BAY 41-8543, $n=6$ ). (B) Representative image of cross-section of the heart stained with Siruis red in SD rats and vehicle- and BAY 41-8543-treated dTCR (left). Perivascular fibrosis area was normalized to vessel media cross-sectional area. Heart sections of vehicle-treated dTCR showed increased cardiac perivascular matrix deposition compared with SD rats; this increase was reduced in dTCR administered BAY 41-8543 ( ${ }^{*} P<0.05,{ }^{* *} P<0.001 ; 1$-way ANOVA with Tukey's post hoc test; data expressed as mean \pm SEM; SD, $n=6$; vehicle, $n=6$; BAY 41-8543, $n=$ 6). (C) Representative image of fibronectin IHC staining in the hearts from SD rats and vehicle- and BAY 41-8543-treated dTCR (left). Interstitial cardiac fibrosis was estimated as fibronectin-positive area per view field. Heart sections of vehicle-treated dTGR showed increased cardiac interstitial fibrosis compared with SD rats; this increase was reduced in dTCR administered BAY 41-8543 ( ${ }^{* *} P<0.01$, ${ }^{* * *} P<0.001 ; 1$-way ANOVA with Tukey's post hoc test; data expressed as mean \pm SEM; SD, $n=6$; vehicle, $n=6$; BAY 41-8543, $n=6$ ). (D) Cardiac connective tissue growth factor (Ctgf) and neutrophil gelatinase-associated lipocalin ( $\mathrm{Ngal}$ ) mRNA expression was significantly higher in vehicle-treated dTCR compared with SD rats. BAY 41-8543 treatment led to a decrease of cardiac Ctgf and $\mathrm{Ngal}$ mRNA expression. mRNA levels of the target genes were normalized for the housekeeping gene $18 \mathrm{~S}\left({ }^{*} P<0.05,{ }^{* *} P<\right.$ 0.01; 1-way ANOVA with Tukey's post hoc test; data expressed as mean \pm SEM; SD, $n=6$; vehicle, $n=8$; BAY 41-8543, $n=8-10$ ).

Perspectives. HFpEF currently has no effective pharmacological treatment. In the dTGR model of experimental HFpEF, we found that BAY 41-8543 yielded a significant reduction in morbidity and mortality. NOsGC stimulation with BAY 41-8543 ameliorated structural remodeling without affecting cardiac hypertrophy, restoring physiological gene expression and cardiac function. Our data suggest that NOsGC stimulators may offer a novel therapeutic treatment option for patients with HFpEF.

\section{Methods}

Animal studies. At the age of 3.5 weeks, age- and body weight-matched male dTGR and nontransgenic SD control rats were randomly assigned to 3 experimental groups: vehicle-treated dTGR (10\% transcutol, 20\% cremophor, 70\% water; $n=13)$, dTGR-treated with $3 \mathrm{mg} / \mathrm{kg} /$ day BAY 41-8543 $(n=12)$, and SD controls 
A

dTGR Vehicle

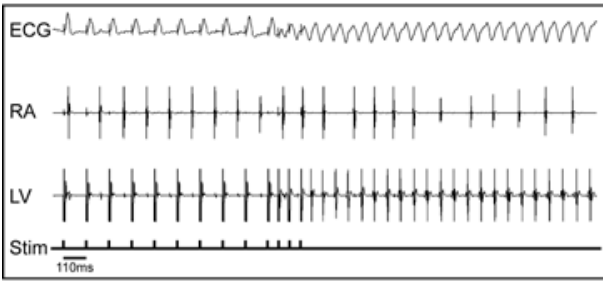

B

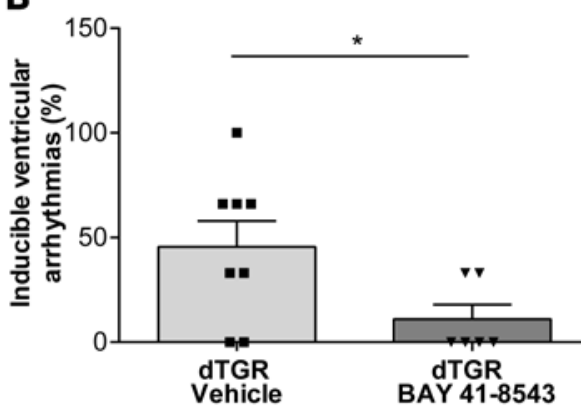

D

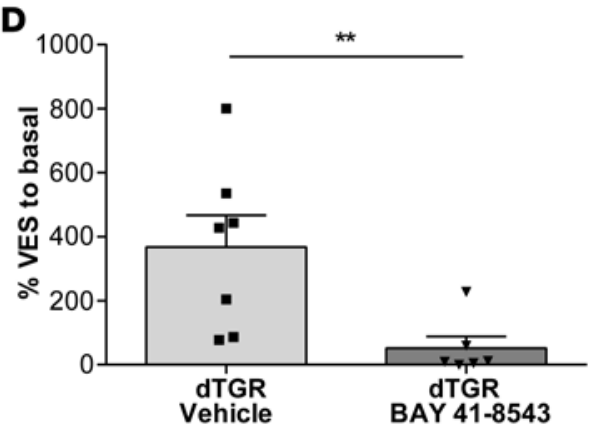

dTGR BAY 41-8543

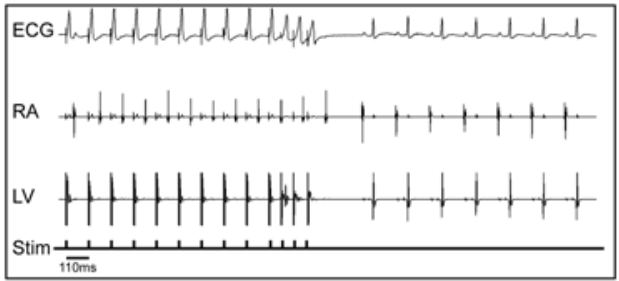

C

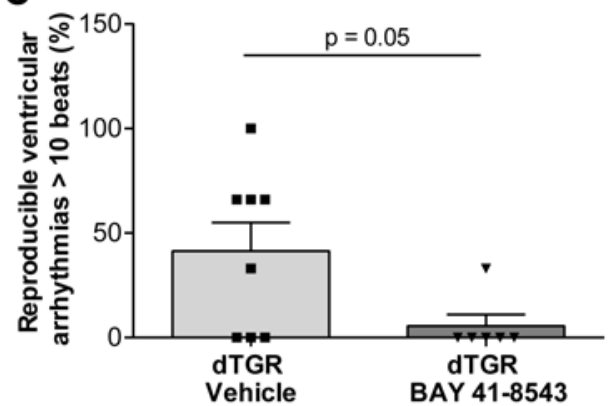

$\mathbf{E}$

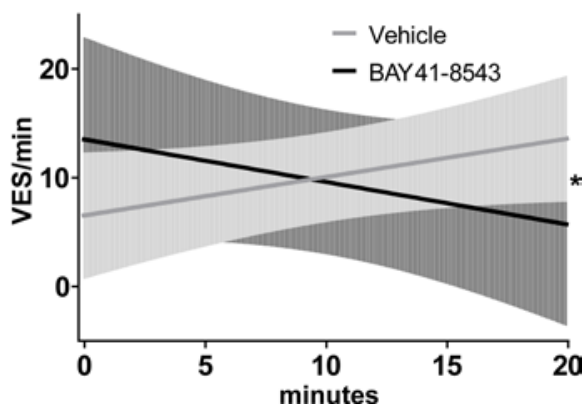

Figure 7. Stimulation of NOsGC improves electrical remodeling and rhythm disturbances. (A) Representative original tracings from in vivo programmed electrical stimulation in vehicle- (left panel) and BAY 41-8543-treated (right panel) dTCR. Surface ECG, right atrial (RA), and left ventricular (LV) electrograms are shown; bottom line shows indicators of delivered electrical stimuli. (B) Inducibility of ventricular arrhythmias by in vivo-programmed electrical stimulation is shown. Given is the percentage of stimulation protocols with inducible arrhythmias from all performed stimulation protocols. Inducibility of ventricular arrhythmias significantly decreased in BAY 41-8543- compared with vehicle-treated dTCR ( $P<0.05 ; 2$-tailed $t$ test; data expressed as mean \pm SEM; vehicle, $n=8$; BAY 41-8543, $n=6$ ). (C) Arrhythmic episodes longer than 10 consecutive ventricular extrasystoles (VES) were reduced in BAY 41-8543- compared with vehicle-treated dTCR (Mann-Whitney $U$ test; data expressed as mean \pm SEM; vehicle, $n=8 ; B A Y 41-8543, n=6$ ). (D) The occurrence of spontaneous VES in isolated Langendorff-perfused dTCR hearts perfused with $3 \mu \mathrm{M}$ BAY 41-8543 or solvent is shown. BAY 41-8543-perfused hearts displayed less VES compared with solvent ${ }^{* *} P<0.01$; Mann-Whitney $U$ test; data expressed as mean \pm SEM; vehicle, $n=7$; BAY 41-8543, $n$ = 6). (E) The occurrence of spontaneous VES over time is reduced in BAY 41-8543-treated isolated Langendorff-perfused dTCR hearts. The slopes for BAY 41-8543- and vehicle-treated dTCR hearts are significantly different ( ${ }^{*} P<0.05$; linear regression; vehicle, $n=7$; BAY $41-8543, n=5$ ).

( $n=10$; Supplemental Figure 2). BAY 41-8543 was used as a tool compound for the sGC stimulator vericiguat since it shares the identical mode of action and has a similar in vitro and in vivo profile.

Treatment was started at the age of 4 weeks by oral gavage once daily until the end of the study at week 7 . Blood pressure was monitored by tail cuff measurements at the age of 5, 6, and 7 weeks. Rats were sacrificed at the age of 7 weeks by decapitation with prior isoflurane anesthesia or due to predefined stopping criteria according with the German/European law for animal protection. The organs were washed with ice-cold saline, blotted dry, and weighed. For gene expression analysis, tissues were snap-frozen in liquid nitrogen and stored at $-80^{\circ} \mathrm{C}$ until further analysis. For IHC and histochemistry, tissues were fixed with formalin and embedded in paraffin. Serum cGMP (IBL RE29075), H-FABP (Hycult Biotech, ELISA HK414, Uden, The Netherlands), BNP (Biotrend RK011-14), and osteopontin (Quantikine ELISA, MOST00, R\&D Systems) were quantified according to the assay manual provided by the manufacturers. Myocardial cGMP was analyzed by a commercial cGMP EIA kit (Cayman Chemical Co.) according to the manufacturer's protocol. Hemodynamic and electrophysiological investigations and echocardiography were performed on a separate set of animals at week 7 . 
In a second study focusing on acute hemodynamic effects of BAY 41-8543, radiotelemetry pressure transducers (TA11PA-C20, Data Sciences International) were implanted in 3.5-week-old dTGRs. At the age of 4 weeks, dTGR were randomly assigned to 2 experimental groups: vehicle $(n=5)$ or $3 \mathrm{mg} / \mathrm{kg} / \mathrm{day}$ BAY 41-8543 $(n=4)$. dTGR received a single oral dosage per day.

Vessel myograph. Mesenteric arteries were removed, quickly transferred to cold $\left(4^{\circ} \mathrm{C}\right)$ oxygenated $(95 \%$ $\mathrm{O}_{2} / 5 \% \mathrm{CO}_{2}$ ) physiological salt solution (PSS) (in mmol/1: $119 \mathrm{NaCl}, 4.7 \mathrm{KCl}, 1.2 \mathrm{KH}_{2} \mathrm{PO}_{4}, 25 \mathrm{NaHCO}_{3}$, $1.2 \mathrm{Mg}_{2} \mathrm{SO}_{4}, 11.1$ glucose, and $1.6 \mathrm{CaCl}_{2}$ ), and dissected into 2-mm rings (47). Each ring was positioned between 2 stainless steel wires $(0.0394 \mathrm{~mm}$ diameter $)$ in a 5-ml PSS-filled organ bath of small vessel myograph (DMT 610M, Danish Myo Technology). The PSS was continuously oxygenated and kept at $37^{\circ} \mathrm{C}$ ( $\mathrm{pH}$ 7.4). Mesenteric rings were placed under a tension equivalent to that generated at 0.9 times the diameter of the vessel at $100 \mathrm{mmHg}(48,49)$. This normalization procedure was performed to obtain the passive diameter of the vessel at $100 \mathrm{mmHg}$. The software LabChart 5 was used for data acquisition and display. The rings were precontracted with $\mathrm{KCl}$ buffer $\left(119 \mathrm{NaCl}, 60 \mathrm{KCl}, 1.2 \mathrm{KH}_{2} \mathrm{PO}_{4}, 25 \mathrm{NaHCO}_{3}, 1.2 \mathrm{Mg}_{2} \mathrm{SO}_{4}\right.$, 11.1 glucose, and $1.6 \mathrm{CaCl}_{2}$ in $\mathrm{mmol} / 1$; all chemicals purchased from MilliporeSigma) and equilibrated until a stable resting tension was acquired; then, the organ bath was washed out with PSS. To test the endothelial function, vessels were precontracted with $1 \mu \mathrm{mol} / 1$ phenylephrine. After all vessel segments had reached a stable contraction plateau, increasing doses of Ach or SNP were administered to the organ baths. Tension was expressed as a percentage of the steady-state tension (100\%) obtained with phenylephrine.

Echocardiography. Transthoracic echocardiography was performed directly before euthanasia at week 6.5 under anesthesia (anesthesia induction in a chamber with $2 \%$, continuation with $1 \%-1.5 \%$ isoflurane) on a tiltable, heated platform to maintain $37^{\circ} \mathrm{C}$ body temperature, executed by a single examiner blinded to the treatment groups ( $n=6-10$ rats per group). Chest hair was gently removed using a clipping machine and depilatory cream. A Visualsonics VEVO2100 imaging system with a $21 \mathrm{MHz}$ transducer (MS250) mounted on an integrated rail system was used, simultaneously recording an electrocardiogram. M-mode and B-mode images were obtained in parasternal short- and long-axis views for measurement of diastolic LV wall thickness (IVSd, LVPWd) and LV systolic function (EF, FS), respectively. The apical 4-chamber view was used to obtain mitral inflow profiles by pulsed wave Doppler (PWD) for estimation of diastolic function (E/A ratio, DTT, IVRT). Diastolic annular velocities $\left(\mathrm{E}^{\prime}, \mathrm{A}^{\prime}\right)$ were captured by tissue Doppler imaging (TDI) at the septal mitral annulus. Images were acquired and stored for offline analysis.

Offline myocardial strain imaging analysis was performed by an investigator unaware of treatments using Visual Sonics Vevo Strain software version 2.2.0 with a speckle-tracking algorithm. B-Mode cine loops were checked for image quality with regard to differentiation of wall borders and absence of artefacts. Strain analysis was performed on 2 consecutive cardiac cycles. The endocardium of the LV was traced manually in parasternal short- and long-axis view in end-diastole. The epicardium was automatically traced by the software, checked, and manually adjusted if necessary for maximum tracking accuracy. Global strain values were obtained from the average of the 6 segments of the LV. LV dyssynchrony was measured using the standard deviation of time-to-peak strain for all 6 segments.

Hemodynamic measurements. For LV PV measurements using the closed-chest approach, rats $(n=6-10$ per group at weeks 6.5) were anesthetized using isoflurane, placed on a heated pad to maintain $37^{\circ} \mathrm{C}$ body temperature, intubated, and ventilated (Rodent Ventilator, 7025, Ugo Basile). A 2-French rat PV conductance catheter (SPR 838 Aria, Millar Instruments) was inserted into the LV via the right carotid artery, guided by online PV signals, and verified by echocardiography. Signals were recorded and analyzed using an MPVS UltraPV system (Millar Instruments), a PowerLab 8/30 recording unit, and LabChart software v8.1.3 (AD Instruments).

In vivo cardiac electrophysiology. To test for the inducibility of ventricular arrhythmias, programmed electrical stimulation was performed in anesthetized, 6.5-week-old dTGR treated with BAY 41-8543 or vehicle. During anesthesia with isoflurane (Univentor 400 anesthesia unit, Agnthos AB), body temperature was kept constant at $37^{\circ} \mathrm{C}$ using an $872 / 3 \mathrm{HF}$ homeothermic blanket control unit (Hugo Sachs Elektronik). We used a digital electrophysiology lab (EP Tracer, CardioTek). A standard limb lead surface ECG was recorded using skin electrodes. Two small octapolar electrophysiology catheters (CIBer mousecath, NuMed) were placed in the right-atrial and left-ventricular cavities via the right jugular vein and the right carotid artery. Programmed ventricular stimulation was performed using a standardized protocol that included trains of 10 basal stimuli (S1) followed by up to 3 extra stimuli (S2-S4), delivered with a coupling interval decreasing in steps of $5 \mathrm{~ms}$ until ventricular refractory was reached. 
The stimulation procedures were repeated at 2 different basal cycle lengths (120 ms, $110 \mathrm{~ms}, 100 \mathrm{~ms})$. Occurrence and duration of inducible arrhythmias were documented. Only stimulation protocols with reproducible ventricular arrhythmias longer than 5 consecutive beats were considered positive. The relative portion of positive protocols from all performed stimulation protocols were compared between the groups.

Langendorff isolated hearts. Seven-week-old dTGRs were killed by decapitation with prior isoflurane anesthesia. Beating hearts were rapidly excised and placed in ice-cold modified Krebs-Henseleit buffer, where the aorta was cannulated. Hearts were immediately connected to a IH-SR Core System (Hugo Sachs Elektronik) and were perfused in a retrograde fashion at constant aortic pressure $(70 \mathrm{mmHg})$ and constant diastolic LV pressure (20 mmHg) with continuously aerated $\left(95 \% \mathrm{O}_{2}\right.$ to $\left.5 \% \mathrm{CO}_{2}\right)$ modified Krebs-Henseleit buffer containing (in mmol/1) $118 \mathrm{NaCl}, 4.7 \mathrm{KCl}, 1.2 \mathrm{MgSO}_{4}, 1.8 \mathrm{CaCl}_{2}, 22.45 \mathrm{NaHCO}_{3}, 0.23 \mathrm{KH}_{2} \mathrm{PO}_{4}$, and 11.1 glucose, at a temperature of $37^{\circ} \mathrm{C}$. The hearts were stabilized for 10 minutes and then treated for 20 minutes with either $3 \mu \mathrm{M}$ BAY $41-8543$ or $0.1 \%$ DMSO as control. Hugo Sachs Elektronik software was used for the data acquisition.

$m R N A$ isolation and quantitative PCR. Total mRNA was isolated from the LV of the heart using QIAzol lysis reagent and Qiagen RNeasy mini kit (Qiagen) with on-column deoxyribonuclease I step (Qiagen) according to the manufacturer's protocol. mRNA $(2 \mu \mathrm{g})$ was reverse transcribed into cDNA using High Capacity cDNA Reverse Transcription Kit (Applied Biosystems). Real-time PCR was detected on ABI 7500 Fast Sequence Detection System (Applied Biosystems) and analyzed by 7500 Fast System Software (Applied Biosystems). Primers and probes (Supplemental Table 1) were designed with Primer Express 3.0 (Applied Biosystems) and synthesized by BioTeZ. Quantitative analysis of target mRNA expression was performed with real-time PCR using the relative standard curve method. The expression level of the target genes was normalized by the expression of the $18 \mathrm{~S}$ housekeeping gene. Samples were run in triplicate, and mean was used for further calculations. The arbitrary units reflect the ratio of the target mRNA concentration divided by the concentration of $18 \mathrm{~S}$ of the same sample.

$I H C$. Paraffin-embedded hearts were cut 4-6 $\mu \mathrm{m}$ thick and stained for collagens using Sirius red. Immunohistological techniques were performed as previously described (28). Heart sections were incubated with primary antibodies against rat monocytes/macrophages (ED-1) (AbD SeroTec, MCA341R), fibronectin (FN) (Abcam, ab23751), wheat germ agglutinin (WGA) (Vector Laboratories, FL1021), Cx43 (Cell Signaling Technology, 3412) and N-cadherin (NCad) (Thermo Fisher Scientific, 33-3900, clone 3B9). Semi-quantitative analyses of IHC and histology were conducted without knowledge of the specific treatment and performed using KS 3003.0 (Zeiss) and Image J software. For the histological and cardiomyocyte analysis, 6 hearts of each group were examined. Ten to 15 different areas of each heart were analyzed. For the determination of the cardiomyocyte size, 100 cardiomyocytes per heart in 10 different areas were evaluated. A mean score for each animal was computed and used to derive a group mean score.

Gene array. Heart samples (SD, $n=8$; dTGR vehicle, $n=8$; and dTGR BAY 41-8543, $n=12$ ) were first homogenized in a Precellys 24 homogenizer $(2.8 \mathrm{~mm}$ reinforced Keramik-Kit/1× 60s at 5,000 rpm), and RNA was isolated using Qiagen RNeasy Mini kits. For microarray analysis, $250 \mathrm{ng}$ of total RNA of each sample was amplified using the Ambion WT Expression Kit (catalog 4411974) and Affymetrix WT Terminal Labeling and Hybridization Kit (catalog 901524) according to the protocol described in User Manual Target Preparation for Gene Chip Whole Transcript (WT) Expression Arrays (part no. [PN] 4425209 Rev. D). Affymetrix Rat Gene 2.1 ST 96-array plates were hybridized with $3 \mu \mathrm{g}$ of fragmented and labeled single stranded cDN (sscDNA), washed, stained, and scanned according to the protocol described in User Manual GeneTitan Instrument User Guide for Expression Arrays Plates (PN 702933 Rev.1) and Affymetrix GeneChip Command Console User's Guide (PN 702569 Rev.9) using Affymetrix GeneTitan platform. Raw chip data (CEL files) were loaded into the Genedata Expressionist Refiner module. Data were condensed, and expression levels for individual chips were calculated after background correction and normalization using the RMA algorithm (50). For data analysis, all chips were shifted to a median expression value of 100 and further corrected by locally weighted scatterplot smoothing (LOWESS) normalization. Microarray data have been uploaded to the NCBI Gene Expression Omnibus (accession number GSE108904).

Statistics. All data are presented as mean \pm SEM. Normal distribution was assessed by Kolmogorov-Smirnov test. Group differences were analyzed by 2-tailed unpaired $t$ test, Mann-Whitney $U$ test, 1 -way ANOVA with Tukey post hoc test, or 2-way ANOVA with Bonferroni post hoc test, as appropriate. Survival 
was examined using a Kaplan-Meier analysis. The ROUT method was performed for outlier identification with an average FDR less than $1 \% . P<0.05$ was considered statistically significant.

Study approval. Local authorities approved the studies (State Office of Health and Social Affairs Berlin; permit no. G0232/09).

\section{Author contributions}

NW and NH designed research studies, conducted experiments, acquired data, analyzed data, and wrote the manuscript. LM, AB, KK, FH, HB, MG, and OS analyzed data. IAS and NR conducted experiments, acquired data, and analyzed data. AH acquired data. DB, JPS, and PS designed research studies and provided reagents. AK, RL, and FS acquired data and analyzed data. FCL wrote the manuscript. DNM and $\mathrm{RD}$ designed research studies, supervised experiments, and wrote the manuscript

\section{Acknowledgments}

The study was funded through a postdoc grant by Bayer AG (to LM). We thank Jutta Meisel, Astrid Schiche, Juliane Anders, and Ute Gerhard for their excellent technical assistance.

Address correspondence to: Ralf Dechend, Experimental and Clinical Research Center, Lindenberger Weg 80, 13125 Berlin, Germany. Phone: 49.30.450540302; Email: ralf.dechend@charite.de.

1. Owan TE, Hodge DO, Herges RM, Jacobsen SJ, Roger VL, Redfield MM. Trends in prevalence and outcome of heart failure with preserved ejection fraction. NEngl J Med. 2006;355 (3):251-259.

2. Borlaug BA, Paulus WJ. Heart failure with preserved ejection fraction: pathophysiology, diagnosis, and treatment. Eur Heart $J$ 2011;32(6):670-679

3. Westermann D, et al. Role of left ventricular stiffness in heart failure with normal ejection fraction. Circulation. 2008;117(16):2051-2060.

4. Borbély A, et al. Cardiomyocyte stiffness in diastolic heart failure. Circulation. 2005;111 (6):774-781.

5. van Heerebeek L, et al. Diastolic stiffness of the failing diabetic heart: importance of fibrosis, advanced glycation end products, and myocyte resting tension. Circulation. 2008;117(1):43-51.

6. Westermann D, et al. Cardiac inflammation contributes to changes in the extracellular matrix in patients with heart failure and normal ejection fraction. Circ Heart Fail. 2011;4 (1):44-52.

7. Ponikowski P, et al. 2016 ESC Guidelines for the diagnosis and treatment of acute and chronic heart failure: The Task Force for the diagnosis and treatment of acute and chronic heart failure of the European Society of Cardiology (ESC). Developed with the special contribution of the Heart Failure Association (HFA) of the ESC. Eur J Heart Fail. 2016;18(8):891-975.

8. Massie BM, et al. Irbesartan in patients with heart failure and preserved ejection fraction. N Engl J Med. 2008;359(23):2456-2467.

9. Redfield MM, et al. Effect of phosphodiesterase-5 inhibition on exercise capacity and clinical status in heart failure with preserved ejection fraction: a randomized clinical trial. JAMA. 2013;309 (12):1268-1277.

10. Cannon PJ. The kidney in heart failure. N Engl J Med. 1977;296(1):26-32.

11. Packer M. Pathophysiology of chronic heart failure. Lancet. 1992;340(8811):88-92.

12. Komajda M, Lam CS. Heart failure with preserved ejection fraction: a clinical dilemma. Eur Heart J. 2014;35(16):1022-1032.

13. Cleland JG, et al. Perindopril for elderly people with chronic heart failure: the PEP-CHF study. The PEP investigators. Eur $J$ Heart Fail. 1999;1(3):211-217.

14. Yusuf S, et al. Effects of candesartan in patients with chronic heart failure and preserved left-ventricular ejection fraction: the CHARM-Preserved Trial. Lancet. 2003;362 (9386):777-781.

15. Pfeffer MA, Pitt B, McKinlay SM. Spironolactone for heart failure with preserved ejection fraction. NEngl J Med. 2014;371(2):181-182.

16. Gu J, et al. Long-term prescription of beta-blocker delays the progression of heart failure with preserved ejection fraction in patients with hypertension: A retrospective observational cohort study. Eur J Prev Cardiol. 2016;23(13):1421-1428.

17. Edelmann F, et al. Tolerability and Feasibility of Beta-Blocker Titration in HFpEF Versus HFrEF: Insights From the CIBISELD Trial. JACC Heart Fail. 2016;4 (2):140-149.

18. Greene SJ, et al. The cGMP signaling pathway as a therapeutic target in heart failure with preserved ejection fraction. $J A m$ Heart Assoc. 2013;2(6):e000536.

19. Pieske B, et al. Rationale and design of the SOluble guanylate Cyclase stimulatoR in heArT failurE Studies (SOCRATES). Eur $J$ Heart Fail. 2014;16 (9):1026-1038.

20. van Heerebeek L, et al. Low myocardial protein kinase G activity in heart failure with preserved ejection fraction. Circulation. 2012;126 (7):830-839

21. Marti CN, Gheorghiade M, Kalogeropoulos AP, Georgiopoulou VV, Quyyumi AA, Butler J. Endothelial dysfunction, arterial stiffness, and heart failure. J Am Coll Cardiol. 2012;60 (16):1455-1469.

22. Buys E, Sips P. New insights into the role of soluble guanylate cyclase in blood pressure regulation. Curr Opin Nephrol Hypertens. 2014;23 (2):135-142.

23. Cawley SM, Kolodziej S, Ichinose F, Brouckaert P, Buys ES, Bloch KD. sGC \{alpha\} 1 mediates the negative inotropic effects of NO in cardiac myocytes independent of changes in calcium handling. Am J Physiol Heart Circ Physiol. $2011 ; 301$ (1):H157-H163.

24. Gheorghiade M, et al. Effect of Vericiguat, a Soluble Guanylate Cyclase Stimulator, on Natriuretic Peptide Levels in Patients 
With Worsening Chronic Heart Failure and Reduced Ejection Fraction: The SOCRATES-REDUCED Randomized Trial. JAMA. 2015;314 (21):2251-2262.

25. Koress C, Swan K, Kadowitz P. Soluble Guanylate Cyclase Stimulators and Activators: Novel Therapies for Pulmonary Vascular Disease or a Different Method of Increasing cGMP? Curr Hypertens Rep. 2016;18(5):42.

26. Conceição G, Heinonen I, Lourenço AP, Duncker DJ, Falcão-Pires I. Animal models of heart failure with preserved ejection fraction. Neth Heart J. 2016;24 (4):275-286.

27. Fischer R, et al. Dietary n-3 polyunsaturated fatty acids and direct renin inhibition improve electrical remodeling in a model of high human renin hypertension. Hypertension. 2008;51 (2):540-546.

28. Haase N, Rugor J, Przybyl L, Qadri F, Müller DN, Dechend R. Relaxin does not improve Angiotensin II-induced target-organ damage. PLoS One. 2014;9 (4):e93743.

29. Luft FC, et al. Hypertension-induced end-organ damage : A new transgenic approach to an old problem. Hypertension. 1999;33 (1 Pt 2):212-218.

30. Mervaala EM, et al. Endothelial dysfunction and xanthine oxidoreductase activity in rats with human renin and angiotensinogen genes. Hypertension. 2001;37 (2 Pt 2):414-418.

31. Wellner M, et al. Cardiac gene expression profile in rats with terminal heart failure and cachexia. Physiol Genomics. 2005;20 (3):256-267.

32. Finckenberg $\mathrm{P}$, et al. Caloric restriction ameliorates angiotensin II-induced mitochondrial remodeling and cardiac hypertrophy. Hypertension. 2012;59 (1):76-84.

33. Hammond J, Balligand JL. Nitric oxide synthase and cyclic GMP signaling in cardiac myocytes: from contractility to remodeling. J Mol Cell Cardiol. 2012;52 (2):330-340.

34. Klotz S, Dickstein ML, Burkhoff D. A computational method of prediction of the end-diastolic pressure-volume relationship by single beat. Nat Protoc. 2007;2 (9):2152-2158.

35. Gallet R, et al. Cardiosphere-derived cells reverse heart failure with preserved ejection fraction (HFpEF) in rats by decreasing fibrosis and inflammation. JACC Basic Transl Sci. 2016;1 (1-2):14-28.

36. Kai H, Kuwahara F, Tokuda K, Imaizumi T. Diastolic dysfunction in hypertensive hearts: roles of perivascular inflammation and reactive myocardial fibrosis. Hypertens Res. 2005;28 (6):483-490.

37. Paulus WJ, Tschöpe C. A novel paradigm for heart failure with preserved ejection fraction: comorbidities drive myocardial dysfunction and remodeling through coronary microvascular endothelial inflammation. J Am Coll Cardiol. 2013;62 (4):263-271.

38. Ahluwalia A, et al. Antiinflammatory activity of soluble guanylate cyclase: cGMP-dependent down-regulation of P-selectin expression and leukocyte recruitment. Proc Natl Acad Sci USA. 2004;101 (5):1386-1391.

39. Zile MR, et al. Myocardial stiffness in patients with heart failure and a preserved ejection fraction: contributions of collagen and titin. Circulation. 2015;131(14):1247-1259.

40. Tarjus A, et al. Neutrophil Gelatinase-Associated Lipocalin, a Novel Mineralocorticoid Biotarget, Mediates Vascular Profibrotic Effects of Mineralocorticoids. Hypertension. 2015;66 (1):158-166.

41. Masuyama $\mathrm{H}$, et al. Soluble guanylate cyclase stimulation on cardiovascular remodeling in angiotensin II-induced hypertensive rats. Hypertension. 2006;48 (5):972-978.

42. Sharkovska Y, et al. Nitric oxide-independent stimulation of soluble guanylate cyclase reduces organ damage in experimental low-renin and high-renin models. J Hypertens. 2010;28 (8):1666-1675.

43. An X, et al. Speckle Tracking Based Strain Analysis Is Sensitive for Early Detection of Pathological Cardiac Hypertrophy. PLoS One. 2016;11 (2):e0149155.

44. Santos AB, et al. Left ventricular dyssynchrony in patients with heart failure and preserved ejection fraction. Eur Heart $J$. 2014;35(1):42-47.

45. Zile MR, et al. Mode of death in patients with heart failure and a preserved ejection fraction: results from the Irbesartan in Heart Failure With Preserved Ejection Fraction Study (I-Preserve) trial. Circulation. 2010;121 (12):1393-1405.

46. Pieske B, et al. Vericiguat in patients with worsening chronic heart failure and preserved ejection fraction: results of the SOluble guanylate Cyclase stimulatoR in heArT failurE patientS with PRESERVED EF (SOCRATES-PRESERVED) study. Eur Heart $J$. 2017;38(15):1119-1127.

47. Schleifenbaum J, et al. Stretch-activation of angiotensin II type 1a receptors contributes to the myogenic response of mouse mesenteric and renal arteries. Circ Res. 2014;115 (2):263-272.

48. Fésüs G, et al. Adiponectin is a novel humoral vasodilator. Cardiovasc Res. 2007;75 (4):719-727.

49. Heinze C, et al. Disruption of vascular Ca2+-activated chloride currents lowers blood pressure. J Clin Invest. $2014 ; 124$ (2):675-686.

50. Irizarry RA, Bolstad BM, Collin F, Cope LM, Hobbs B, Speed TP. Summaries of Affymetrix GeneChip probe level data. Nucleic Acids Res. 2003;31 (4):e15. 